\title{
Vortices and Their Relation to Ring Currents and Magnetic Moments in Nanographenes in High Magnetic Field
}

\author{
S. Compernolle,* L. F. Chibotaru, and A. Ceulemans \\ Laboratorium voor kwantumchemie, and INPAC-Institute for Nanoscale Physics and Chemistry, \\ Katholieke Universiteit Leuven, Celestijnenlaan 200F, B-3001 Heverlee, Belgium
}

Received: June 23, 2006; In Final Form: July 28, 2006

\begin{abstract}
Much attention has been paid to the role of vortices in the magnetic response properties of superconductors, but less so for molecular systems. Here we present a theoretical analysis on nanographenes subject to a strong homogeneous magnetic field. The analysis is based on the simple Hückel-London model, for which we derive the topological definition of vorticity. The results are confirmed by a more elaborate model that includes nonnearest neighbor interaction, the explicit presence of nuclei and all terms due to the magnetic field. We find that due to frontier orbital intersections, large changes in magnetic dipole moments occur. Orbital energy minima and maxima can be related to change of vortex patterns with flux.
\end{abstract}

\section{Introduction}

In general, a complex one-particle wave function $\psi(\mathbf{r})$ can be written as

$$
\psi(\mathbf{r})=|\psi(\mathbf{r})| \exp (i \theta(\mathbf{r}))
$$

with $\theta(\mathbf{r})$ the phase of the wave function. Although both the wave function and its absolute value should be continuous and single valued, the same is not true for the phase, which is only defined modulo $2 \pi$. Therefore, the integral of the gradient of phase around a closed loop $C$ need not vanish and will in general be equal to

$$
\oint_{\mathrm{C}} \nabla \theta \cdot \mathrm{dl}=2 \pi v
$$

with $\mathbf{I}$ the vector tangential to the contour $C$, in the clockwise direction, and $v$ an integer. Note that $\nabla \theta$ is a measure of the velocity. A nonzero value for $v$ implies the presence of one or more vortices inside the contour $C$. At the position of a vortex, the wave function vanishes, i.e., $\psi(\mathbf{r})=|\psi(\mathbf{r})|=0 . v$ is the sum of the winding numbers (positive for a clockwise circulation) of the vortices inside $C$ and is called the vorticity. Vortices arise, for example, in superconductors of the second type in the presence of a magnetic field. ${ }^{1,2}$ The general features of vortex patterns and their evolution with the applied magnetic field have been recently investigated for mesoscopic superconductors ${ }^{3,4}$ and normal quantum dots. ${ }^{5}$ The fundamental concept of vortices in electronic wave functions was investigated a long time ago. ${ }^{6-10}$ Also the creation of vortices in molecular orbitals by magnetic fields and its connection with orbital ring currents has been considered, ${ }^{11,12}$ and the specific case of benzene in a low magnetic field was worked out. ${ }^{13}$

Ring currents in conjugated carbon systems have been since long a subject of intense theoretical research, since they play an important role in the interpretation of magnetic properties such as nuclear magnetic shieldings, the magnetic dipole moment, and susceptibility. In general, the focus has been on the properties of rather small hydrocarbons at low or moderate magnetic fields. An exception is ref 14 where benzene and cyclooctatetraene were theoretically investigated under a field of $25 \mathrm{~T}$. But even for this relatively high field, the nonlinear contribution to the current density is still small.
The ability to synthesize large hydrocarbons or "nanographenes" in a controlled way has improved remarkably. ${ }^{15-17}$ This opens the way to investigate new quantum effects. In particular, we have reported in a recent communication that sudden variations of the magnetic dipole moment of nanographenes at high magnetic field occur due to intersections of the frontier molecular orbitals. ${ }^{18}$ In this work we provide a full account of these results and extend the study to cyclic conjugated $\pi$-electron systems. Our analysis is based on the simple topological Hückel-London (HL) model. HL is often the method of choice to handle large graphitic systems, since it produces reliable results with a minimal effort. Many quantities can be made "topological"; e.g., the current density can be resolved over bond currents. ${ }^{19,20}$ However, this method does not strictly define a continuous wave function, which is necessary if the location of a vortex is to be known. Therefore, we have derived the HL topological definition of vorticity and established its link with ring currents (see Appendix A).

Other limitations of HL include lack of explicit consideration of the nuclei, neglect of overlap, nonnearest neighbor interactions, and the variation of the vector potential over distances of the order of the bond length. ${ }^{21}$ Therefore, we have also developed a more sophisticated method, the screened nuclei approximation with magnetic field (SNM) (see Appendix B), which confirms qualitatively the HL results and allows for a more detailed analysis, since the position of the vortices can exactly be traced, as opposed to the HL vorticity.

\section{Method}

2.1. Huickel-London Formalism. 2.1.1. Hamiltonian. For the derivation of the HL method, we refer to ref 21 . This theory only considers $\pi$ electrons and nearest-neighbors interactions and neglects any overlap between orbitals. For a homogeneous magnetic field taken in the $z$ direction, a matrix element between two nearest neighbors is approximated as

$$
H_{\mathrm{st}}=-|\beta| \exp \left(i \frac{2 \pi}{\phi_{0}} B \int_{\mathrm{st}}\right)=-|\beta| \exp \left(i \frac{2 \pi \phi_{\mathrm{st}}}{\phi_{0}}\right)
$$

with $\beta \approx-2.5 \mathrm{eV}$ the transfer integral between nearest neighbor atomic orbitals $\mathrm{s}$ and $\mathrm{t}, B$ the strength of the magnetic field, 


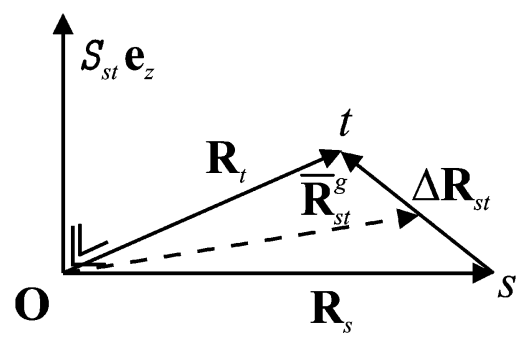

Figure 1. Schematic representation of the vectors $\mathbf{R}_{\mathrm{s}}, \mathbf{R}_{\mathrm{t}}$, denoting atomic positions, their geometrical center $\overline{\mathbf{R}}_{\mathrm{st}}^{\mathrm{g}}$, and their difference $\Delta \boldsymbol{R}_{\mathrm{st}}$. The area of the triangle formed by the origin $\mathbf{O}$ and the atoms $\mathrm{s}$, $\mathrm{t}$ is equal to $\int_{\text {st }}$, to be counted positive when $\Delta \boldsymbol{R}_{\text {st }}$ is anticlockwise oriented with respect to the origin, and negative otherwise.

$\phi_{0}=$ hcle the magnetic flux quantum, and $\int_{\mathrm{st}}=(1 / 2) \mathbf{e}_{z}$. $\left(\mathbf{R}_{\mathrm{s}} \times \mathbf{R}_{\mathrm{t}}\right)=(1 / 2)\left(\Delta \mathbf{R}_{\mathrm{st}} \times \mathbf{e}_{z}\right) \cdot \overline{\mathbf{R}}_{\mathrm{st}}^{\mathrm{g}}$ the signed algebraic area formed by the origin and the atoms $\mathrm{s}$ and $\mathrm{t}$, where $\overline{\mathbf{R}}_{\mathrm{st}}^{\mathrm{g}}=$ $\left(\mathbf{R}_{\mathrm{s}}+\mathbf{R}_{\mathrm{t}}\right) / 2$ is the geometrical center between atoms $\mathrm{s}$ and $\mathrm{t}$ and $\Delta \boldsymbol{R}_{\mathrm{st}}=\mathbf{R}_{\mathrm{t}}-\mathbf{R}_{\mathrm{s}}$ (Figure 1). Note that since we are considering flat systems, the atoms s and t are located in the $x y$ plane. $\phi_{\mathrm{st}}=B \int_{\mathrm{st}}$ is the magnetic flux through the triangle $\int_{\mathrm{st}}$. $\int_{\text {st }}$ is positive when $\Delta \boldsymbol{R}_{\text {st }}$ is anticlockwise oriented with respect to the origin and negative otherwise.

2.1.2. Bond Currents and Bond Order. Following the bond current formalism presented in refs 19 and 20, a bond current $J_{\text {st }}^{\mathrm{b}}$ and a MO bond current $J_{1, \mathrm{st}}^{\mathrm{b}}$ between two atomic orbitals are equal to

$$
\begin{gathered}
J_{\mathrm{st}}^{\mathrm{b}}=\sum_{l} n_{\mathrm{l}} J_{1, \mathrm{st}}^{\mathrm{b}} \\
J_{1, \mathrm{st}}^{\mathrm{b}}=-\frac{2 e|\beta|}{\hbar} \operatorname{Im}\left[c_{1, \mathrm{~s}}^{*} c_{1, \mathrm{t}} \exp \left(i \frac{2 \pi \phi_{\mathrm{st}}}{\phi_{0}}\right)\right] \\
=-\frac{2 e|\beta|}{\hbar} \sqrt{q_{1, \mathrm{~s}} q_{1, \mathrm{t}}} \sin \left(\Delta \gamma_{1, \mathrm{st}}+\frac{2 \pi \phi_{\mathrm{st}}}{\phi_{0}}\right)
\end{gathered}
$$

with $n_{1}$ the occupation number of $\mathrm{MO} \psi_{1}, c_{1, \mathrm{t}}$ the coefficient of the atomic orbital on site $\mathrm{t}, \Delta \gamma_{1, \mathrm{st}}=\operatorname{Im}\left[\ln \left(c_{1, \mathrm{t}} / c_{1, \mathrm{~s}}\right)\right]$ the phase difference between coefficients $c_{\mathrm{l}, \mathrm{s}}$ and $c_{\mathrm{l}, \mathrm{t}}$, and $q_{1, \mathrm{t}}=\left|c_{1, \mathrm{t}}\right|^{2}$ the MO charge on atom $t$. When $J_{\text {st }}^{\mathrm{b}}>0$ the conventional current is directed from $s$ to $t$, the flow of electrons from $t$ to $s$. Note that in our pictures we always depict the flow of the electrons (Figure 2a). The summation runs over all occupied orbitals. The bond current is closely related to the bond order $\mathscr{B}_{\mathrm{st}}$, which can also be split up into MO contributions $\mathscr{B}_{1, \mathrm{st}}$

$$
\begin{gathered}
\mathscr{B}_{\mathrm{st}}=\sum_{l} n_{1} \mathscr{B}_{1, \mathrm{st}} \\
\mathscr{B}_{1, \mathrm{st}}=\operatorname{Re}\left[c_{1, \mathrm{~s}}^{*} c_{1, \mathrm{t}} \exp \left(i \frac{2 \pi \phi_{\mathrm{st}}}{\phi_{0}}\right)\right] \\
=\sqrt{q_{1, \mathrm{~s}} q_{1, \mathrm{t}}} \cos \left(\Delta \gamma_{1, \mathrm{st}}+\frac{2 \pi \phi_{\mathrm{st}}}{\phi_{0}}\right)
\end{gathered}
$$

so for a given MO charge of the atoms $\mathrm{s}$ and $\mathrm{t}, J_{1, \mathrm{st}}^{\mathrm{b}}=0$ means a minimal or maximal MO bond order. Note that the following relation holds for bond currents

$$
\sum_{t}^{\langle s\rangle} J_{\mathrm{st}}^{\mathrm{b}}=\sum_{t}^{\langle s\rangle} J_{1, \mathrm{st}}^{\mathrm{b}}=0
$$

where $\langle s\rangle$ indicates that the summation runs only over the nearest neighbors of atom $\mathrm{s}$. This is the $\mathrm{HL}$ variant of charge

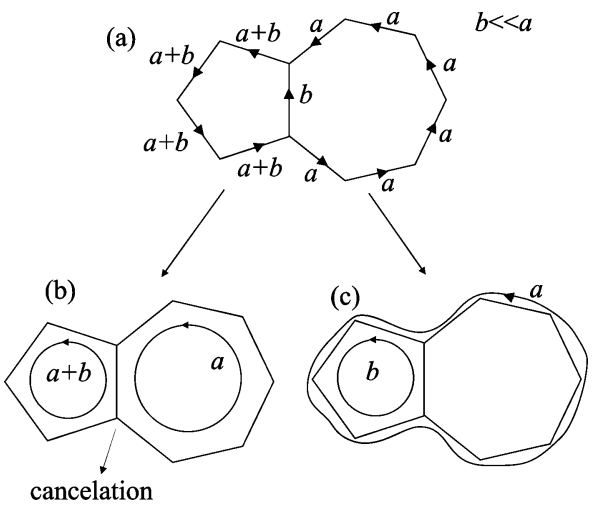

Figure 2. HL currents for azulene. The arrows indicate the flow of the electrons. (a) Bond current picture. Note that there are only two independent bond currents, $a, b$. At small field, $b \ll a .^{24}$ (b) Face current picture. In this view, there are two large diamagnetic ring currents in both faces. As a consequence, there is a large cancelation of current where the two faces share an edge. (c) Constructive ring current picture. Imposing that a ring current must follow the direction of the arrows leads to a unique solution for azulene: a diamagnetic ring current around the perimeter of the molecule and another diamagnetic one in the pentagon. No cancelation of current occurs.

conservation of a stationary system. Consider a system with $N_{\mathrm{v}}$ atoms (or vertexes), $N_{\mathrm{e}}$ bonds (or edges), and $N_{\mathrm{f}}$ faces. There are of course $N_{\mathrm{v}}$ equations such as (8), but in general only $N_{\mathrm{v}}-1$ are independent. This means that, eventual symmetry not taken into account, the number of independent bond currents $N_{\text {ic }}$ for a flat system is equal to

$$
N_{\text {ic }}=N_{\mathrm{e}}-\left(N_{\mathrm{v}}-1\right)=N_{\mathrm{f}}
$$

This number corresponds to the number of independent "ring closure bonds" on the spanning tree of the molecular graph. ${ }^{22}$ Equation 9 also reflects the Euler relationship for a planar molecule. ${ }^{23}$ Symmetry can reduce this number. If the current density distribution of a system is known, its magnetic dipole moment $m_{z}$ can be calculated, which can be split up in a sum over orbital contributions $m_{z, 1}$. In the HL model these quantities can be related to the bond currents ${ }^{20}$

$$
\begin{gathered}
m_{z}=-\frac{\mathrm{d} E}{\mathrm{~d} B}=\frac{1}{c} \sum_{\langle\mathrm{st}\rangle} J_{\mathrm{st}}^{\mathrm{b}} \int_{\mathrm{st}}=\sum_{l} n_{1} m_{z, 1} \\
m_{z, \mathrm{l}}=-\frac{\mathrm{d} \epsilon_{1}}{\mathrm{~d} B}=\frac{1}{c} \sum_{\langle\mathrm{st}\rangle} J_{1, \mathrm{st}}^{\mathrm{b}} \rho_{\mathrm{st}}
\end{gathered}
$$

where the sum runs over distinct bond pairs. $\epsilon_{1}$ denotes the orbital energy of MO $\psi_{1}$.

2.1.3. Ring Currents. In the description above, the total current and MO current picture is decomposed in a set of bond currents $J_{\mathrm{st}}^{\mathrm{b}}$. An alternative view is a decomposition in a set of ring currents $J_{\mathrm{r}}^{\mathrm{R}}{ }^{24}$ In this view, one considers a set of closed paths of edges and to each such path a current is associated. For a given ring current, each edge of the path carries the same current in the same direction. Summing all ring currents must of course give back the original set of bond currents. Since there are $N_{\text {ic }}$ independent bond currents, the number of independent ring currents is also $N_{\text {ic }}$. From this viewpoint, the magnetic dipole moment becomes ${ }^{24}$

$$
m_{z}=\sum_{r} m_{z, \mathrm{r}}^{\mathrm{R}}, \quad \text { with } \quad m_{z, \mathrm{r}}^{\mathrm{R}}=\frac{1}{c} J_{\mathrm{r}}^{\mathrm{R}} \int_{\mathrm{r}}
$$




$$
m_{z, 1}=\sum_{r} m_{z, 1, \mathrm{r}}^{\mathrm{R}}, \quad \text { with } \quad m_{z, 1, \mathrm{r}}^{\mathrm{R}}=\frac{1}{c} J_{1, \mathrm{r}}^{\mathrm{R}} \rho_{\mathrm{r}}
$$

with $J_{\mathrm{r}}^{\mathrm{R}}\left(J_{1, \mathrm{r}}^{\mathrm{R}}\right)$ the ring current (MO ring current) around the path $r, \mathcal{S}_{\mathrm{r}}$, the surface of the path, and $m_{z, \mathrm{r}}^{\mathrm{R}}\left(m_{z, \mathrm{l}, \mathrm{r}}^{\mathrm{R}}\right)$ the dipole moment (MO dipole moment) due to the ring current. If we take $\int_{\mathrm{r}}$ positive by convention, then $J_{\mathrm{r}}, J_{1, \mathrm{r}}, m_{z, \mathrm{r}}^{\mathrm{R}}$, and $m_{z, \mathrm{r}, \mathrm{r}}^{\mathrm{R}}$ are positive for an anticlockwise ring current circulation (flow of conventional current) and negative for a clockwise one. The advantage of this picture is that the individual ring currents $J_{\mathrm{r}}^{\mathrm{R}}, J_{1, \mathrm{r}}^{\mathrm{R}}$ look more physical as they obey all (8) as opposed to the individual bond currents $J_{\mathrm{st}}^{\mathrm{b}}, J_{1, \mathrm{st}}^{\mathrm{b}}$. The drawback is that for larger systems there are many ways to decompose the current in ring currents, depending on the choice of the set of closed paths of edges. One could for example choose the faces as paths, but this choice is arbitrary (Figure 2b).

Second, cancelation of current can occur where two ring currents meet, a feature which has no physical meaning. However, the number of possibilities can be severely diminished if one builds in a natural restriction: the direction of the ring current on each of its edges must be equal to that of the bond currents on those edges. As a consequence, when two ring currents share an edge, they must have the same direction on that edge. Current cancelations will therefore never occur. We call such a set of ring currents a set of constructive ring currents. Although the set of constructive ring currents does not have to be unique in general, it is often the case for small systems (Figure 2c).

As we will prove later on, there is a general relation between constructive ring currents and vorticity, which is not true for a general ring current decomposition.

2.1.4. Bond Phases and Vorticity. For a one-electron wave function, such as a MO $\psi_{1}$, vorticity of a closed contour $C$ can be defined as

$$
v=\frac{1}{2 \pi} \oint_{\mathrm{C}} \nabla \theta_{1} \cdot \mathrm{dl}
$$

with the gradient of phase $\nabla \theta_{1}$ defined as

$$
\nabla \theta_{1}(\mathbf{r})=\frac{1}{\left|\psi_{1}(\mathbf{r})\right|^{2}} \operatorname{Im}\left[\psi_{1}^{*}(\mathbf{r}) \nabla \psi_{1}(\mathbf{r})\right]=\operatorname{Im}\left[\nabla \ln \psi_{1}(\mathbf{r})\right]
$$

Within HL theory, when the contour is chosen along a closed path of edges, the vorticity becomes

$$
\begin{gathered}
v=\sum_{\mathrm{st}}^{C}-\frac{\Delta \tilde{\gamma}_{1, \mathrm{st}}}{2 \pi} \\
\Delta \tilde{\gamma}_{1, \mathrm{st}}=\Delta \gamma_{1, \mathrm{st}}-2 \pi \operatorname{Int}\left[\frac{\Delta \gamma_{1, \mathrm{st}}}{2 \pi}+\frac{\phi_{\mathrm{st}}}{\phi_{0}}\right]
\end{gathered}
$$

where the sum runs over the edges st of the closed path $C$ and $\Delta \tilde{\gamma}_{1, \mathrm{st}}$ is the integrated phase gradient over the edge st, shortly called the bond phase. Int $[x]$ is the integer closest to $x$. The proof of eq 16 is given in Appendix A. Special care must be taken when a vortex is located exactly on an edge (see Appendix A).

With this topological HL definition for vorticity, the highest "vorticity resolution" one can obtain of a MO is that of face vorticities: one knows only the sum of the winding numbers of the vortices inside a face, not the nature and exact position of each vortex inside that face. But since within HL theory the smallest possible circulations of current are around faces, a higher resolution is unnecessary.

We have tested eq 16 by checking it numerically with eq 2 on HL MOs of several nanographenes, where the atomic orbital coefficients were coupled to $2 \mathrm{p}_{z}$ type Slater orbitals. The two methods agree perfectly in the limit of a high extinction coefficient of the Slater orbitals (i.e., the limit of high localization). Of course, eq 16 is much easier to evaluate.

It follows directly from eqs 5 and 17 that

$$
J_{1, \mathrm{st}}^{\mathrm{b}}=-\frac{2 e|\beta|}{\hbar} \sqrt{q_{1, \mathrm{~s}} q_{1, \mathrm{t}}} \sin \left(\Delta \tilde{\gamma}_{1, \mathrm{st}}+\frac{2 \pi \phi_{\mathrm{st}}}{\phi_{0}}\right)
$$

Since $\Delta \tilde{\gamma}_{1, \mathrm{st}}+\left(2 \pi \phi_{\mathrm{st}} / \phi_{0}\right)$ is always within the interval $[-\pi, \pi]$, the sign of $J_{1, \mathrm{st}}^{\mathrm{b}}$ is opposite to that of $\Delta \tilde{\gamma}_{1, \mathrm{st}}+\left(2 \pi \phi_{\mathrm{st}} / \phi_{0}\right)$

$$
\begin{aligned}
& 0 \leq \Delta \tilde{\gamma}_{1, \mathrm{st}}+2 \pi \frac{\phi_{\mathrm{st}}}{\phi_{0}} \Longrightarrow J_{1, \mathrm{st}}^{\mathrm{b}} \leq 0 \\
& \Delta \tilde{\gamma}_{1, \mathrm{st}}+2 \pi \frac{\phi_{\mathrm{st}}}{\phi_{0}} \leq 0 \Longrightarrow J_{1, \mathrm{st}}^{\mathrm{b}} \geq 0
\end{aligned}
$$

\section{Benzene}

The archetype of the conjugated hydrocarbon is benzene. We derive here the general formula for cyclic conjugated $\pi$-electron systems ( $N_{\mathrm{v}}$ atoms) with rotational symmetry $C_{N_{\mathrm{v}}}$, of which benzene is a special case $\left(N_{\mathrm{v}}=6\right)$. The MOs obey $C_{N_{\mathrm{v}}} \psi_{k}=$ $\mathrm{e}^{-2 \pi i k / N_{\mathrm{v}}} \psi_{k}$. Within HL theory there is only one MO per rotational symmetry number $k$, so we can use it as a label for MOs and MO properties. HL predicts perfectly oscillating orbital energies and orbital magnetic dipole moments with flux $\phi$ with period $N_{\mathrm{v}} \phi_{0}$

$$
\begin{gathered}
\epsilon_{k}=-2|\beta| \cos \left(\frac{2 \pi}{N_{\mathrm{v}}}\left(-k+\frac{\phi}{\phi_{0}}\right)\right) \\
m_{z, k}=-\frac{2 S_{N_{\mathrm{v}}}|\beta|}{\phi_{0}} \frac{2 \pi}{N_{\mathrm{v}}} \sin \left(\frac{2 \pi}{N_{\mathrm{v}}}\left(-k+\frac{\phi}{\phi_{0}}\right)\right) \\
k=0, \pm 1, \pm 2, \ldots, \frac{N_{\mathrm{v}}}{2} \text { for } N_{\mathrm{v}} \text { even } \\
k=0, \pm 1, \pm 2, \ldots, \pm \frac{N_{\mathrm{v}}-1}{2} \text { for } N_{\mathrm{v}} \text { odd }
\end{gathered}
$$

with $S_{N_{\mathrm{v}}}$ the surface of the ring:

$$
S_{N_{\mathrm{v}}}=N_{\mathrm{v}} \frac{r_{\mathrm{cc}}^{2}}{4} \cot \frac{\pi}{N_{\mathrm{v}}}
$$

At any flux, HL predicts mirror symmetry of the orbital energies around energy zero. For benzene we have $\epsilon_{0}=-\epsilon_{3}, \epsilon_{1}=-\epsilon_{-2}$, $\epsilon_{2}=-\epsilon_{-1}$. In agreement with the HL model, the orbital energies calculated by the SNM method display an oscillating pattern (Figure 3a). The exact periodicity, and the perfect symmetry around the nonbonding energy is however broken by the inclusion of non-nearest neighbors. In addition one can see a parabolic rise of background in energy and a decreasing difference between the orbital energies, which is also not accounted for by the approximate HL model. At zero field, only $\psi_{0}$ and $\psi_{ \pm 1}$ are occupied orbitals. 
(a)

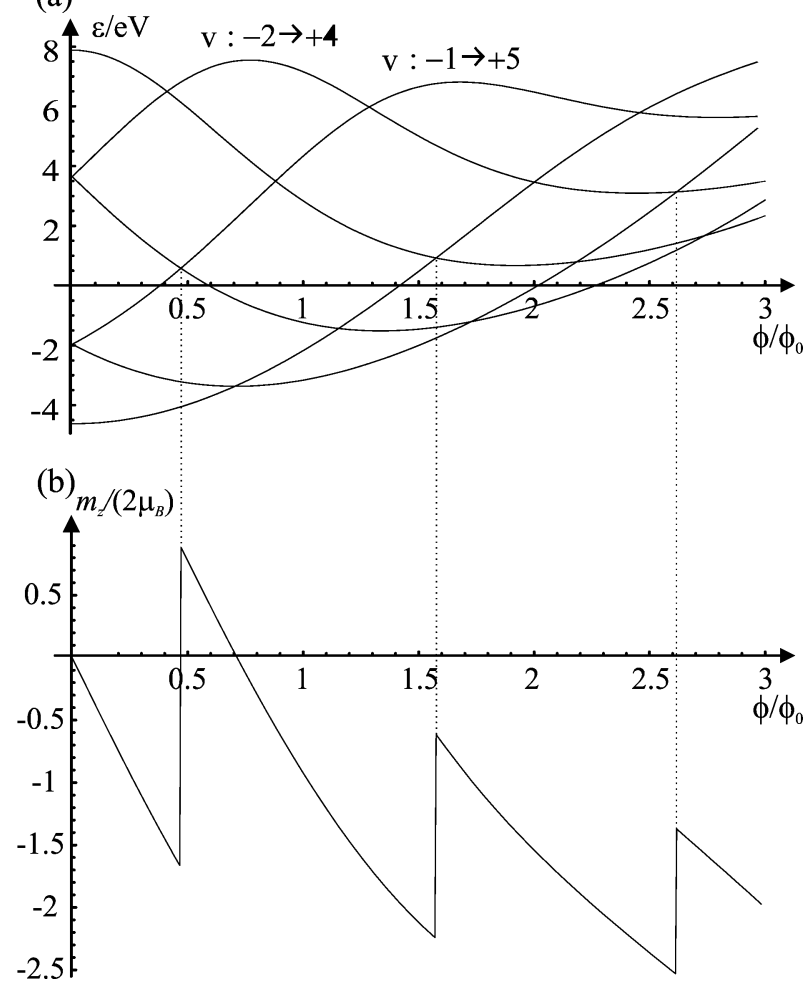

Figure 3. (a) Variation of orbital energies of benzene with an applied perpendicular magnetic field, as calculated by the SNM method. Orbital energies are given in $\mathrm{eV}$ and the magnetic flux in units of the flux quantum. Only the three lowest levels are occupied. The change of vorticity of $\psi_{-2}$ and of $\psi_{-1}$ at the maximum of orbital energy is also indicated. (b) Magnetic dipole moment of benzene vs magnetic flux. The magnetic dipole moment is given in units of $1 / 2 \mathrm{Bohr}$ magneton. The crossing points of orbital energies between an occupied and unoccupied MO correspond to discrete jumps in the magnetic dipole moment.

As we deal with a single ring system, there is only one ring current, equal to each of the $N_{\mathrm{v}}$ bond currents: $J_{1}^{\mathrm{R}}=J_{\mathrm{st}}^{\mathrm{b}}=J .^{25}$

$$
\begin{gathered}
J=\sum_{k} n_{k} J_{k} \\
J_{k}=-\frac{2 e|\beta|}{\hbar} \frac{1}{N_{\mathrm{v}}} \sin \left(\frac{2 \pi}{N_{\mathrm{v}}}\left(-k+\frac{\phi}{\phi_{0}}\right)\right)
\end{gathered}
$$

When the external field is directed to the positive $z$-direction, a rising orbital energy corresponds to a clockwise ( $y$ to $x$ ) or diamagnetic orbital ring current $\left(J_{\mathrm{k}}^{\mathrm{R}}<0\right)$ and a lowering energy to an anticlockwise ( $x$ to $y$ ) or paramagnetic ring current $\left(J_{\mathrm{k}}^{\mathrm{R}}>0\right)$. The bond phase for any edge of the orbital $\psi_{k}$ is equal to

$$
\Delta \tilde{\gamma}_{k, \mathrm{st}}=-\frac{2 \pi k}{N_{\mathrm{v}}}-2 \pi \operatorname{Int}\left[\frac{1}{N_{\mathrm{v}}}\left(-k+\frac{\phi}{\phi_{0}}\right)\right]
$$

The vorticity of the orbital $\psi_{k}$ at any flux in the HL model is equal to

$$
v_{k}=-\frac{N_{\mathrm{v}}}{2 \pi} \Delta \tilde{\gamma}_{k, \mathrm{st}}=k+N_{\mathrm{v}} \operatorname{Int}\left[\frac{1}{N_{\mathrm{v}}}\left(-k+\frac{\phi}{\phi_{0}}\right)\right]
$$

Since all bond currents are equivalent, it follows from eq 19 that the direction of the ring current of a MO is determined by both the vorticity of the MO and the flux through the molecule

$$
\begin{gathered}
\text { diamagnetic } \Leftrightarrow J_{k}<0 \Leftrightarrow \frac{\phi}{\phi_{0}}-v_{k}>0 \\
\text { paramagnetic } \Leftrightarrow J_{k}>0 \Leftrightarrow \frac{\phi}{\phi_{0}}-v_{k}<0
\end{gathered}
$$

Let us now consider the special case of benzene in four different situations: (i) the low flux regime, (ii) crossings between HOMO and LUMO, (iii) orbital energy maxima, (iv) orbital energy minima.

At the low flux regime $0<\phi / \phi_{0} \ll 1$, we get

$$
v_{k}=k, \quad k=0, \pm 1, \pm 2,3
$$

Note that for $-1 \ll \phi / \phi_{0}<0$ (the magnetic field in the opposite direction), $v_{3}=-3$ while the other vorticities remain the same. Except for $\psi_{0}$, the direction of all ring currents is determined by the vortex in the center. In the MO $\psi_{0}$ no vortex is present and hence the ring current is diamagnetic. Since at low flux

$$
\begin{gathered}
J_{k} \approx-\frac{2 e|\beta|}{\hbar} \frac{1}{6}\left(-\sin \left(\frac{k \pi}{3}\right)+\frac{\pi}{3} \frac{\phi}{\phi_{0}} \cos \left(\frac{k \pi}{3}\right)\right) \\
\frac{\phi}{\phi_{0}} \rightarrow 0
\end{gathered}
$$

the total ring current is - as is well-known ${ }^{24}$-diamagnetic at low flux and as a consequence the magnetic dipole moment is negative

$$
\begin{gathered}
J=2\left(J_{0}+J_{1}+J_{-1}\right) \approx-2 \frac{2 e|\beta|}{\hbar} \frac{1}{6} \frac{2 \pi}{3} \frac{\phi}{\phi_{0}} \\
m_{z} \approx-2 \frac{|\beta| S_{6}}{\phi_{0}} \frac{4 \pi^{2}}{9} \frac{\phi}{\phi_{0}}
\end{gathered}
$$

where the factor 2 is to account for spin degeneracy. In nice agreement with HL theory, SNM calculations show that $\psi_{0}$, $\psi_{-1}, \psi_{-2}$ are characterized by diamagnetic ring currents and $\psi_{1}, \psi_{2}, \psi_{3}$ by paramagnetic ones. ${ }^{18}$

In the high-flux regime crossings between the orbital energies of an occupied and unoccupied MO occur. HL predicts such crossings at $\phi / \phi_{0}=(2 q+1) / 2$ with $q$ an integer. Because of the symmetry between the bonding and antibonding orbital energies, these crossings will always occur between $\psi_{-1}$ and $\psi_{2}, \psi_{0}$ and $\psi_{3}, \psi_{1}$ and $\psi_{-2}$. Let $\phi_{k \rightarrow k^{\prime}}$ be the field where the orbital energy $\epsilon_{k}$ of an occupied MO and the orbital energy $\epsilon_{k^{\prime}}$ of a unoccupied MO become equal. Hence

$$
\begin{aligned}
& \phi<\phi_{k \rightarrow k^{\prime}} \Longrightarrow n_{k}=2, \quad n_{k^{\prime}}=0 \\
& \phi>\phi_{k \rightarrow k^{\prime}} \Longrightarrow n_{k}=0, \quad n_{k^{\prime}}=2
\end{aligned}
$$

Of course there is a small transient region around $\phi_{k \rightarrow k^{\prime}}$ where the system should be described by a triplet state $\left(n_{k}=n_{k^{\prime}}=1\right)$ instead of a singlet due to the interaction between the electrons in these orbitals (Hund's rule). If this effect is not taken into 


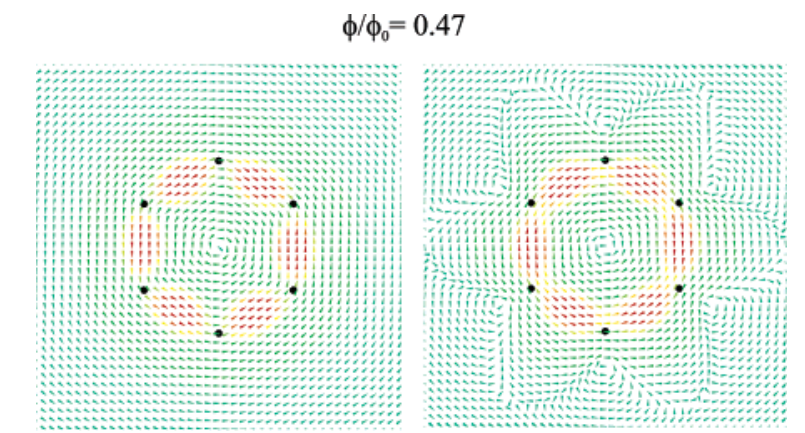

Figure 4. SNM current density maps of the MOs $\psi_{-1}$ (left) and $\psi_{2}$ (right) of benzene at $B=B_{-1 \rightarrow 2} \approx 0.157$ au, calculated in a plane of $2 a_{0} / Z_{\text {eff }}$ above the nuclei (at this height the $2 \mathrm{p}_{z}$ orbitals have their maximal value). Blue arrows denote low and red high values of current density. The black dots denote the positions of the atoms.

account, the magnetic dipole moment changes instantaneously from negative to positive at a frontier orbital intersection

$$
m_{z}^{+}-m_{z}^{-}=2\left(m_{z, k^{\prime}}-m_{z, k}\right)=2 \frac{1}{c}\left(J_{k^{\prime}}-J_{k}\right) S_{\mathrm{h}}=2 \frac{|\beta| S_{6}}{\phi_{0}} \frac{4 \pi}{3}
$$

$$
m_{z}^{+}=-m_{z}^{-}=2 \frac{|\beta| S_{6}}{\phi_{0}} \frac{2 \pi}{3}
$$

with $m_{z}{ }^{+}, m_{z}{ }^{-}$the magnetic dipole moment just after and just before the frontier orbital intersection, respectively. For benzene, this jump corresponds to $2 \times 2.29 \mu_{\mathrm{B}}$-where $\mu_{\mathrm{B}}$ is the Bohr magneton-for a value of $\beta=-2.5 \mathrm{eV}$.

The SNM method predicts the first three crossings at fluxes $\phi_{-1 \rightarrow 2} \approx 0.47 \phi_{0}, \phi_{0 \rightarrow 3} \approx 1.58 \phi_{0}$, and $\phi_{1 \rightarrow-2} \approx 2.61 \phi_{0}$, which compares well with the HL values. As opposed to HL theory, the jumps diminish in size at higher fields: the first three jumps are approximately $2 \times 2.5 \mu_{\mathrm{B}}, 2 \times 1.5 \mu_{\mathrm{B}}$, and $2 \times 1 \mu_{\mathrm{B}}$ (Figure $3 b)$. This decrease is caused by the exponential decay with flux of the off-diagonal Hamiltonian matrix elements in the SNM method (see Appendix B.2), whereas the transfer integral $\beta$ in $\mathrm{HL}$ is assumed constant. Also, the jumps at higher flux are not sufficient to change $m_{z}$ from negative to positive: the local diamagnetic atomic currents - neglected in HL theory-become of similar importance as the global ring current. In Figure 4 the SNM current densities of $\psi_{-1}$ and $\psi_{2}$ are shown at $\phi_{-1 \rightarrow 2}$. The large increase of $m_{z}$ is caused by the replacement of the originally diamagnetic ring current of $\psi_{-1}$ by the paramagnetic ring current of $\psi_{2}$. The other jumps in $m_{z}$ have a similar explanation. Allowing the system to be a triplet around $\phi_{k \rightarrow k^{\prime}}$ would make the jumps of $m_{z}$ less steep but would not remove them. Let us mention that also the magnetic susceptibility $\chi_{\|}$ changes steeply at the fields $\phi_{k \rightarrow k^{\prime}}$.

When the energy of an orbital goes through a maximum, this indicates that the orbital ring current switches from diamagnetic to paramagnetic. Within HL theory, an orbital energy maximum for $\epsilon_{k}$ will occur each time $\phi / \phi_{0}=3(2 q+1)+k$, with $q$ an integer. It follows from eq 25 that at these fluxes the vorticity of the MO increases by 6 and becomes bigger than $\phi / \phi_{0}$ (eq 26). At the same time, following eq 7 the bonds become totally antibonding which means a point of zero density is present at the edges.

The interpretation is that at these fluxes, six new vortices enter the MO through the edges, causing the vorticity to increase by 6 and forcing the ring current to change direction. To show that this interpretation is the correct one, we compare these results with the SNM calculations. HL predicts the first

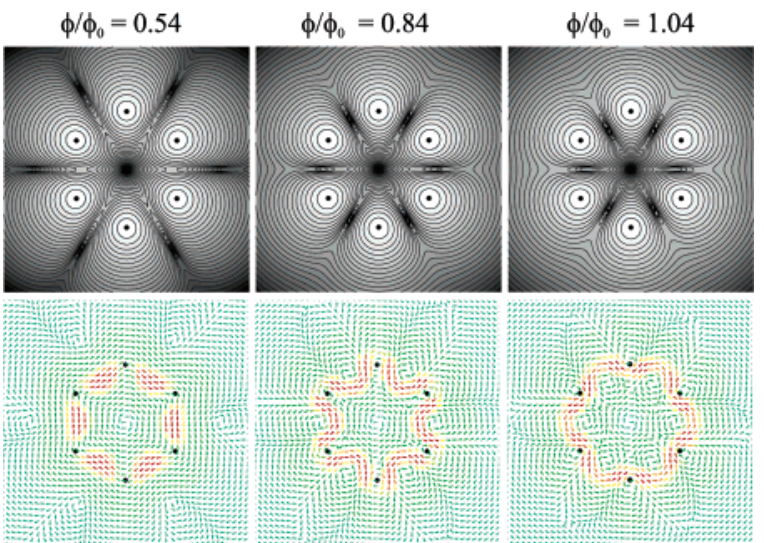

Figure 5. SNM density and current density maps of the MO $\psi_{-2}$ of benzene before, at, and after the orbital energy maximum at $B \approx 0.26$ $\mathrm{au}$, calculated in a plane of $2 a_{0} / Z_{\text {eff }}$ above the nuclei. For the current density maps the same conventions are followed as for Figure 4, while for the density maps white regions correspond to high density and black to low density. Six vortices come from infinity and cross the edges. After their crossing the direction of the ring current has changed from diatropic to paratropic.

maximum at nonzero flux for the $\psi_{-2}$ orbital at $\phi / \phi_{0}=1$, while SNM predicts it at $\phi / \phi_{0} \approx 0.8$. Also the other maxima are estimated at slightly lower flux by SNM compared to HL. Figure 5 shows the SNM density and current density maps of $\psi_{-2}$ before, at, and after the maximum.

Six new vortices-with $v=1$ and coming from infinitycross the bonds, thereby forcing the ring current to change direction. The vorticity of $\psi_{-2}$ is now 4 . The change of vorticity of $\psi_{-2}$ has no immediate effect on the system since it is not an occupied orbital at this field. But its importance is revealed at $\phi=\phi_{1 \rightarrow-2}$ where $\psi_{-2}$ becomes an occupied orbital. The same phenomenon of six new incoming vortices crossing the edges also occurs at the other orbital energy maxima. Note that also the orbital energy maximum at $\phi / \phi_{0}=0$ for $\epsilon_{3}$ can be interpreted in this way (vorticity rises from -3 to 3 ), although the entry of the six vortices cannot be visualized; one could equivalently speak of the entry of six antivortices when going from positive to negative flux.

Finally we discuss the orbital energy minima. HL theory predicts these minima at $\phi / \phi_{0}=6 q+k$. Following eqs 23 and 7 , the HL orbital bond current between two nearest neighbors is then zero, but as opposed to the case of the orbital energy maxima, the bond order is maximal. The vorticity does not change at this flux (eq 25). The ring current reversal is explained by the flux becoming dominant over the vorticity (eq 26). The first orbital energy minimum at nonzero flux is for the $\psi_{1}$ orbital at $\phi / \phi_{0}=1$ according to $\mathrm{HL}$ and at $\phi / \phi_{0} \approx 0.8$ according to SNM. Also the other orbital energy minima predicted by SNM are shifted to lower fluxes compared to HL. Figure 6 shows the SNM density and current density of $\psi_{1}$ before, at, and after the minimum.

It can be seen from the density plots that indeed no vortex motion is visible during the process. The ring current reversal can be interpreted as the diamagnetic circulation due to the magnetic field becoming dominant over the paramagnetic circulation due to the central vortex. The other orbital energy minima have a similar explanation. The above analysis for benzene can easily be extended to other ring sizes.

\section{Cyclic Conjugated $\pi$-Electron Systems}

Since for benzene $\phi_{0}$ corresponds to a magnetic field of $4 \times$ $10^{4} \mathrm{~T}$, it is clear that the phenomena described above cannot 


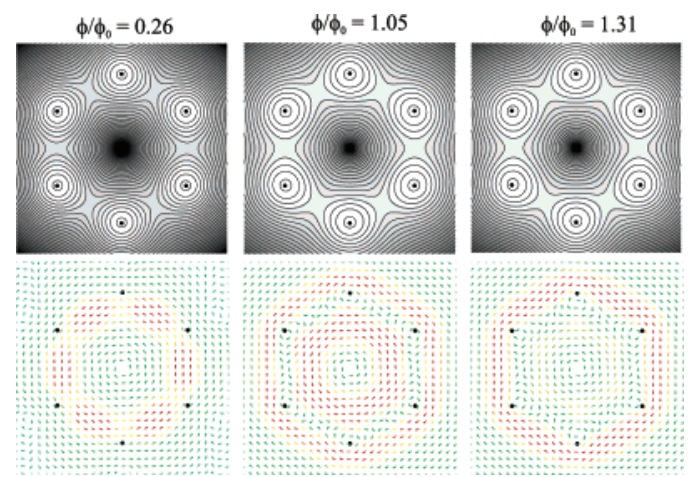

Figure 6. SNM density and current density plots before, at, and after the first orbital minimum of $\psi_{1}$. The same conventions are followed as for Figure 5.

be achieved with a laboratory field, which can maximally reach about $10^{2} \mathrm{~T}$. One way around this is to increase the length of the circular chain, such that the high flux can be reached at lower field. Equation 32 can easily be extended for any circular chain with $N_{\mathrm{v}}=4 q+2$ atoms ( $q$ integer): frontier orbital intersections occur still at $\phi / \phi_{0}=(2 q+1) / 2$ and the jumps in magnetic dipole moment are then

$$
\begin{aligned}
m_{z}^{+}-m_{z}^{-}=2 \frac{|\beta| S_{N_{\mathrm{v}}}}{\phi_{0}} \frac{8 \pi}{N_{\mathrm{v}}}=2 \frac{|\beta| r_{\mathrm{cc}}^{2}}{\phi_{0}} 2 \pi \cot \frac{\pi}{N_{\mathrm{v}}} \approx \\
2 \frac{|\beta| r_{\mathrm{cc}}{ }^{2}}{\phi_{0}} 2 N_{\mathrm{v}}
\end{aligned}
$$

i.e., they increase linearly with the number of atoms. The first jump will occur at a field of

$$
B c^{-1}=\frac{1}{2} \frac{h}{e} \frac{1}{N_{\mathrm{v}} \frac{r_{\mathrm{cc}}{ }^{2}}{4} \cot \frac{\pi}{N_{\mathrm{v}}}} \approx 2 \frac{h}{e} \frac{\pi}{N_{\mathrm{v}}{ }^{2} r_{\mathrm{cc}}{ }^{2}}
$$

which drops below $50 \mathrm{~T}$ for a chain of 162 atoms.

\section{Nanographenes}

Another way to get high flux for relatively low field is to consider large pieces of graphene, the so-called nanographenes. The properties of finite nanographenes will depend on the nature of their edges. Both straight zigzag and armchair edges have been observed in bulk pieces of graphene. ${ }^{26}$ But "bottom up" synthesized nanographenes have predominantly armchair edges, ${ }^{15,27}$ although also structures with partial zigzag periphery have been made. ${ }^{28}$ This is in agreement with theoretical predictions: nanographenes with armchair edges are fully benzenoid and therefore more stable. ${ }^{29} \mathrm{We}$ have performed a series of calculations on hexagonal nanographenes with straight armchair edges and of different sizes. Following the terminology of ref 30, they are symbolized as $\operatorname{Ph}(L)$ where $L$ is the number of layers (Figure 7a). The numbers of atoms (or vertexes), $N_{\mathrm{v}}$, bonds (or edges), $N_{\mathrm{e}}$, and hexagons (or faces), $N_{\mathrm{f}}$, of these systems are equal to

$$
\begin{gathered}
N_{\mathrm{v}}=6\left(1+3 L+3 L^{2}\right) \\
N_{\mathrm{e}}=6\left(1+\frac{1}{2}\left(7 L+9 L^{2}\right)\right) \\
N_{\mathrm{f}}=1+3 L+9 L^{2}
\end{gathered}
$$
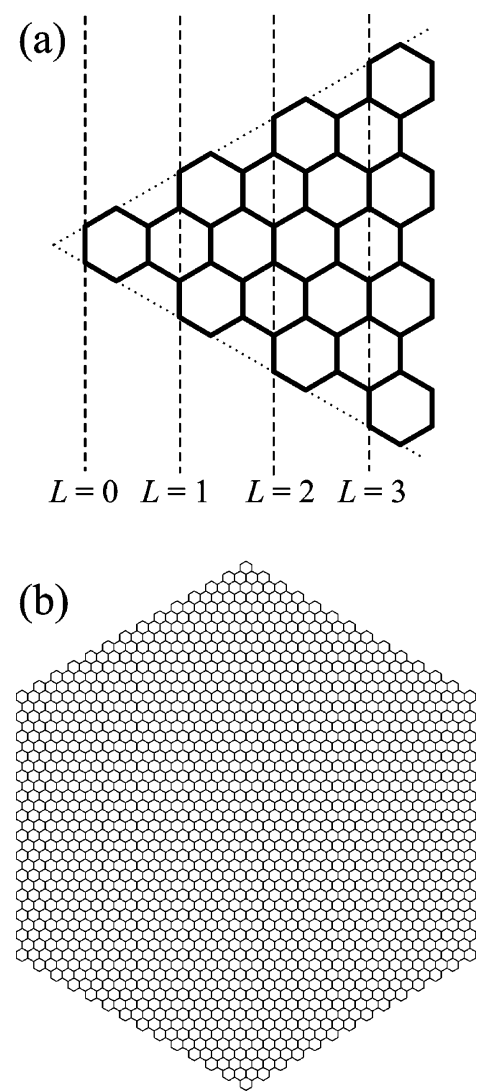

Figure 7. (a) Graphical scheme for the construction of a $D_{6 h}$ nanographene with armchair edges $\operatorname{Ph}(L)$ (only one rotational subunit is shown). The dashed cutting lines determine $L$, the number of layers of the nanographene. (b) A nanographene with $L=13$.

where $L=0$ corresponds to the case of benzene. Note that for planar systems, Euler's theorem takes the following form

$$
N_{\mathrm{v}}-N_{\mathrm{e}}+N_{\mathrm{f}}=1
$$

The largest molecule we investigated, $\mathrm{Ph}(13)$, has 3282 atoms and 1561 hexagons (Figure $7 \mathrm{~b}$ ). The symmetry of these systems is $D_{6 h}$, but the magnetic field, perpendicular to the plane of the molecule, will reduce this to $C_{6 h}$. Actually the flow of the current density is antisymmetric with respect to the vertical mirror planes of the original $D_{6 h}$ symmetry group because of time reversal symmetry. Taking the symmetry into account from the $C_{6}$ axis and the antisymmetry of the vertical mirror planes, the number of independent bond currents is equal to

$$
N_{\text {ic }}= \begin{cases}1+\frac{3}{4} L(L+2) & L \text { even } \\ \frac{3}{4}(L+1)^{2} & L \text { odd }\end{cases}
$$

The orbital energies and MOs can be characterized by an integer rotational symmetry number $k$

$$
\begin{gathered}
H \psi_{k, j}=\epsilon_{k} \psi_{k, j} \\
\hat{C}_{6} \psi_{k, j}=\exp \left(-i \frac{2 \pi k}{6}\right) \psi_{k, j} \\
k=-2, \ldots, 3, \quad j=1, \ldots, N
\end{gathered}
$$

with $\hat{C}_{6}$ the rotation about the central 6-fold axis and $j$ the orbital number within the symmetry group $k$ counted from the lowest energy. 
(a) $\varepsilon / \beta$

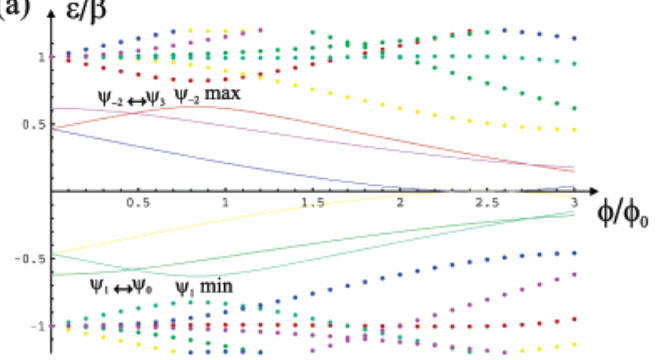

(b) $\varepsilon / \beta$

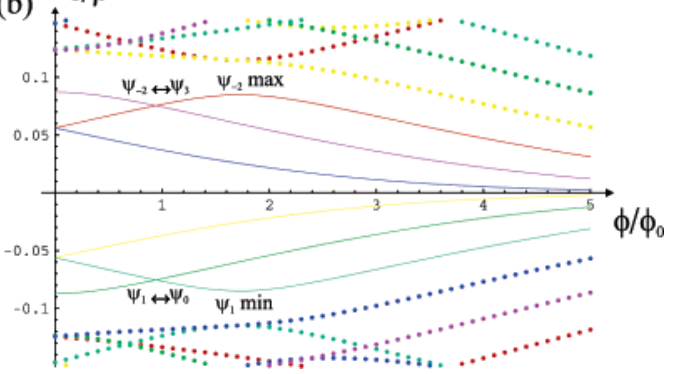

Figure 8. (a) and (b) HL orbital energies vs flux for the $\mathrm{Ph}(1)$ (a) system and the $\mathrm{Ph}(13)(\mathrm{b})$. The central six orbital energies are given in full lines and the others in dotted lines. Indicated features for both plots are $\psi_{0} \leftrightarrow \psi_{1}$ and $\psi_{-2} \leftrightarrow \psi_{3}$ intersections, a minimum (maximum) for $\psi_{1}\left(\psi_{-2}\right)$. There are no intersections of other MOs with the central six.

For the SNM calculations the bond lengths were fixed at the value of graphite: $r_{\mathrm{cc}}=1.42 \AA$.

All the systems we investigated are closed-shell at zero field. The HOMO-LUMO gap decreases slowly and monotonically with size, a feature which was described already by Hückel calculations. ${ }^{31}$ The symmetries of the frontier orbitals of the series have always the same order: HOMO $-1 k=0$, HOMO (degenerate) $k= \pm 1$, LUMO (degenerate) $k= \pm 2, \mathrm{LUMO}+1$ $k=3$. We found that these six MOs do not intersect with other MOs. Since we focus on these six MOs, the subscript $j$ is dropped in $\psi_{k, j}$. Note that these MOs look qualitatively similar for different sizes of the nanographene. In Figure 8a the HL frontier orbital levels from $\mathrm{HOMO}-1$ to $\mathrm{LUMO}+1$ versus flux are given for the $\mathrm{Ph}(1)$ system and in Figure 8b for the $\mathrm{Ph}(13)$ system. Apart from a rescaling, the qualitative features of both plots - and for all $\mathrm{Ph}(L)$ systems with intermediate size-are the same: (i) orbital intersections between $\psi_{0}(\mathrm{HOMO}-1)$ and $\psi_{1}$ (HOMO) and between $\psi_{-2}$ (LUMO) and $\psi_{3}(\mathrm{LUMO}+1)$ at relatively low flux; (ii) an orbital minimum (maximum) at somewhat higher flux for the $\psi_{1}\left(\psi_{-2}\right)$ orbital due to an avoided crossing with a lower (higher) orbital with the same symmetry; (iii) an intersection between $\psi_{-1}$ (HOMO) and $\psi_{2}$ (LUMO) at much higher flux. Figure 9a suggests that the $\psi_{0} \leftrightarrow \psi_{1}$ and $\psi_{-2} \leftrightarrow \psi_{3}$ orbital intersections move asymptotically toward $\phi / \phi_{0}=1$ when the size of the system is increased (Figure 9a).

Also the position of the orbital minimum (maximum) of the $\psi_{1}\left(\psi_{-2}\right)$ moves slowly toward higher fluxes when the size of the system is increased. The HOMO-LUMO $\psi_{-1} \leftrightarrow \psi_{2}$ intersections on the other hand move toward much higher fluxes, and more importantly, the slopes of the intersecting orbital energies are very small. Hence as opposed to the case of benzene, a HOMO-LUMO intersection will not give rise to a large change in magnetic dipole moment. Therefore it is clearly more interesting to investigate the HOMO-LUMO intersections of the $\mathrm{Ph}(L)^{4+}$ cation or the $\mathrm{Ph}(L)^{4-}$ anion (corresponding to the HOMO-1-HOMO and the LUMO-LUMO+1 intersections of the neutral molecule) than the HOMO-LUMO intersections of the neutral structure. (a)

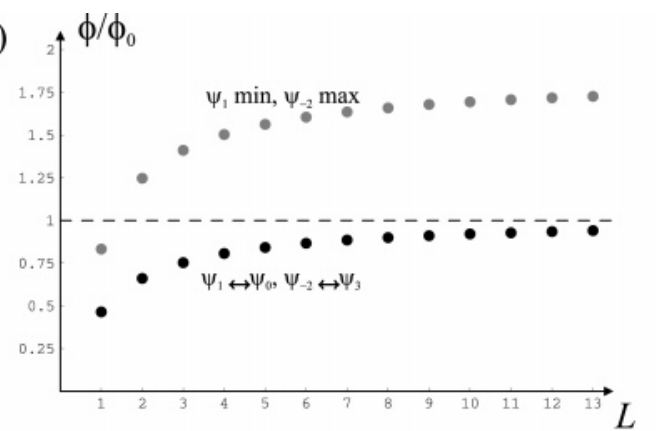

(b)

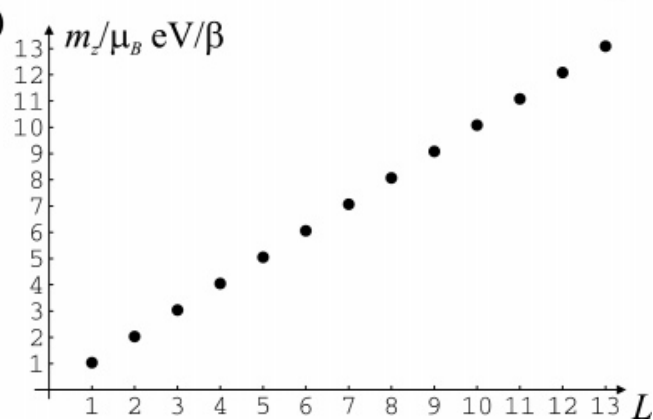

Figure 9. (a) Black dots: flux for which the $\psi_{0} \leftrightarrow \psi_{1}$ and $\psi_{-2} \leftrightarrow \psi_{3}$ intersections occur vs the number of layers. Gray dots: flux for which the minimum (maximum) for $\psi_{1}\left(\psi_{-2}\right)$ occurs vs the number of layers. (b) Jump of the magnetic dipole moment vs the number of layers.

The size of the jump of the magnetic dipole moment at the frontier orbital intersections, both for the $\operatorname{Ph}(L)^{4+}$ cation and the $\mathrm{Ph}(L)^{4-}$ anion, increases linearly with $L$ (Figure $9 \mathrm{~b}$ ). For the $\mathrm{Ph}(13)$ molecule, HL theory predicts these intersections at $\phi / \phi_{0} \approx 0.94$, corresponding to a high but achievable magnetic field of $47 \mathrm{~T}$. The magnetic dipole moment of the $\mathrm{Ph}(13)^{4+}$ cation and the $\mathrm{Ph}(13)^{4-}$ anion changes with $\Delta m_{z}=2 \times$ $0.0383\left(1 / \phi_{0}\right) N_{\mathrm{f}} S_{6}|\beta|$, corresponding to not less than $2 \times 33 \mu_{\mathrm{B}}$.

Using the SNM method gives very similar results. Thus, SNM predicts for the $\mathrm{Ph}(13)^{4+}$ nanographene a HOMO-LUMO intersection at $45 \mathrm{~T}$ ( $\psi_{0} \leftrightarrow \psi_{1}$ intersection), and for the $\mathrm{Ph}(13)^{4-}$ nanographene at $53 \mathrm{~T}\left(\psi_{-2} / \psi_{3}\right.$ intersections $)$. The jumps in magnetic dipole moment at these intersections are $2 \times 16.6 \mu_{\mathrm{B}}$ for the $\mathrm{Ph}(13)^{4+}$ cation and $2 \times 37.5 \mu_{\mathrm{B}}$ for the $\mathrm{Ph}(13)^{4-}$ anion. ${ }^{18}$

The current distribution can be decomposed into a set of constructive ring currents. Similarly to the single ring system, each ring current must obey the condition

$$
\begin{gathered}
\text { diamagnetic } \Leftrightarrow J_{k, \mathrm{r}}^{\mathrm{R}}<0 \Leftrightarrow \frac{\phi_{\mathrm{r}}}{\phi_{0}}-v_{k, \mathrm{r}}>0 \\
\text { paramagnetic } \Leftrightarrow J_{\mathrm{k}, \mathrm{r}}^{\mathrm{R}}>0 \Leftrightarrow \frac{\phi_{\mathrm{r}}}{\phi_{0}}-v_{\mathrm{k}, \mathrm{r}}<0
\end{gathered}
$$

where both the ring current $J_{k, \mathrm{r}}^{\mathrm{R}}$ and the vorticity $v_{k, \mathrm{r}}$ are taken along the ring $r$. This follows directly from the fact that on each edge, $\Delta \tilde{\gamma}_{\mathrm{k}, \mathrm{st}}+2 \pi\left(\phi_{\mathrm{st}} / \phi_{0}\right)$ and $J_{k, \mathrm{st}}^{\mathrm{b}}$ must have opposite sign and that a constructive ring current $J_{k, \mathrm{r}}^{\mathrm{R}}$ always follows the direction of the bond currents. It is important to realize that eq 43 is not generally true for ring current sets that are not constructive, such as a set of currents along the hexagonal faces.

The intersecting orbitals are very similar for all $L$. Let us focus on the $\psi_{-2} \leftrightarrow \psi_{3}$ intersections. The $\psi_{-2}$ orbital has vorticity -2 on all Clar hexagons and +1 on all other hexagons (Figure 10a). We found that at the intersection, all ring currents of the $\psi_{-2}$ orbital are diamagnetic. The most important ring 


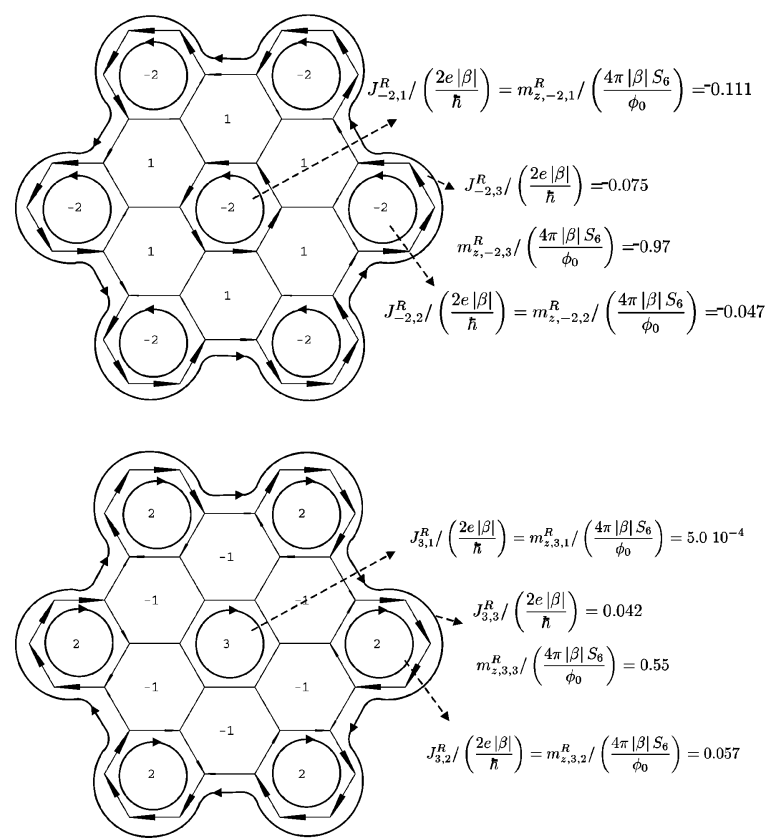

Figure 10. Face vorticities, bond currents, and constructive ring currents of $\mathrm{Ph}(1)$ for $\psi_{-2}$ (a) and $\psi_{3}$ (b) at the $\psi_{-2} \leftrightarrow \psi_{3}$ intersection.

currents are first ring currents around individual Clar hexagons and second currents flowing around the center and with different diameters. The largest ring current is located on the outer edges of the molecule. The contribution from the ring currents on the Clar hexagons to the magnetic dipole moment is relatively unimportant compared to the one flowing around the center since the last group encloses larger areas. This last group encircle zones with large negative vorticities. The contribution of the flux $\phi_{\mathrm{r}} / \phi_{0}$ in eq 43 is for all ring currents smaller compared to the vorticity; hence it is the vorticity that determines the direction of the ring current.

The ring current profile of the $\psi_{3}$ orbital is quite similar, except that all ring currents are now paramagnetic and that the Clar hexagons have vorticity +2 and all other hexagons vorticity -1 (Figure 10b), except for the central Clar hexagon, which has vorticity +3 .

Each time a bond current changes direction, the set of constructive ring currents necessarily changes. An example is given for the orbital maximum of $\psi_{-2}$ of $\mathrm{Ph}(1)$ (Figure 11). From eq 40 we find that there are only three independent bond currents, hence also three independent ring currents. For $\mathrm{Ph}(1)$ the set of constructive ring currents is unique. Before the orbital maximum, all ring currents are diamagnetic, one at the central hexagon, six symmetry related at the corner Clar hexagons, and one around the perimeter of the molecule (Figure 11a). A first change happens when six symmetry related bond currents change direction while the corresponding bond order goes through a maximum. The diamagnetic ring current around the perimeter is now replaced by a paramagnetic ring current that excludes the corner Clar hexagons. The orbital maximum then occurs when the magnetic dipole moment of this paramagnetic ring current becomes of the same size as the sum of the other diamagnetic ring currents. A second change of the ring current set occurs when the vorticity of the corner Clar hexagons changes from -2 to +1 . By comparison with SNM calculations it is found that this vorticity change is caused by a vortex entry of three vortices with winding number +1 in each corner Clar hexagon. As a consequence, the diamagnetic ring currents in the corner Clar hexagons is replaced by a second paramagnetic ring current around the perimeter of the molecule. This shows
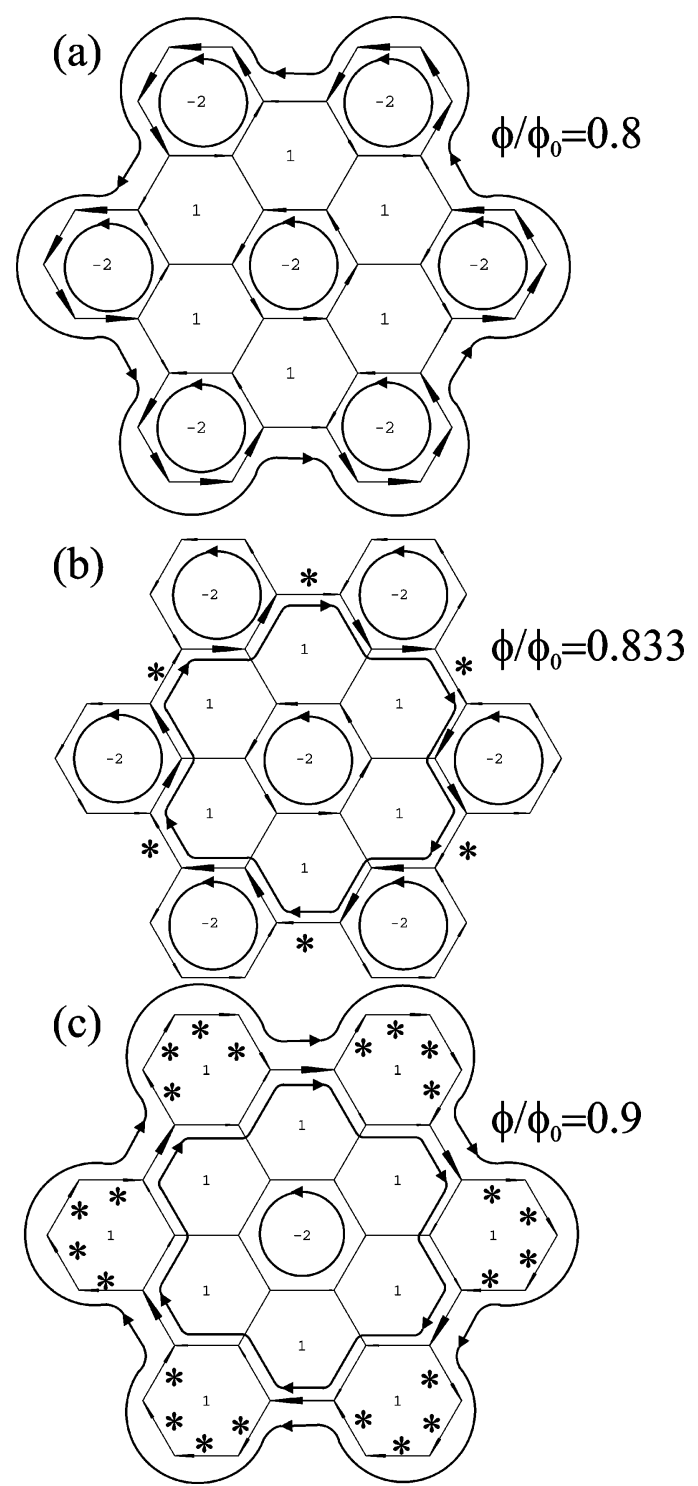

Figure 11. Face vorticities, bond currents, and constructive ring currents for the orbital maximum of $\psi_{-2}$ of $\mathrm{Ph}(1)$, (a) before, (b) at, and (c) after the avoided crossing. The ${ }^{*}$ label shows which bond currents have changed direction compared with the previous plot.

how the entry or exit of vortices have an impact on the MO ring currents. Let us finally mention that also the orbital minimum of $\psi_{1}$ is associated with the motion of vortices.

\section{Discussion and Conclusions}

We have developed a formalism based on HL theory to calculate vorticities of molecular orbitals and made the connection with constructive ring currents. With this method we have analyzed benzene, cyclic conjugated $\pi$-electron systems, and nanographenes with armchair edges $(\mathrm{Ph}(L))$. The results are in good qualitative agreement with the more advanced SNM method.

The frontier orbital intersection of the $\mathrm{Ph}(L)^{4+}$ and the $\mathrm{Ph}(L)^{4-}$ structures lead to large abrupt changes in the magnetic dipole moment $m_{z}$. This jump of $m_{z}$ will occur at a lower field when the size of the system is increased; for $L=13$ it is at around $50 \mathrm{~T}$, which is within reach of present laboratory magnets. The size of the jump increases linearly with $L$. Around MO minima or maxima, motion of vortices in the MOs leads to a change of the ring currents. 
Acknowledgment. Financial support from the Belgian Government through the Concerted Action Scheme (GOA), from the KULeuven Research Council (BOF), and the National Science Foundation (FWO-Vlaanderen) is gratefully acknowledged.

\section{A. Derivation of Topological Vorticity}

In general, when $\psi_{n}(\mathbf{r})$ is known, $\nabla \theta_{n}(\mathbf{r})$ can be calculated numerically. The problem for the HL method is that formally $\psi_{n}$ is expressed as a linear combination of atomic orbitals

$$
\begin{gathered}
\psi_{n}(\mathbf{r})=\sum_{j} c_{n, j} \varphi^{\mathrm{B}}\left(\mathbf{r}-\mathbf{R}_{j}\right) \\
\varphi^{\mathrm{B}}\left(\mathbf{r}-\mathbf{R}_{j}\right)=\exp \left(-i \frac{2 \pi}{\phi_{0}} \mathbf{A}\left(\mathbf{R}_{j}\right) \cdot \mathbf{r}\right) \varphi^{0}\left(\mathbf{r}-\mathbf{R}_{j}\right)
\end{gathered}
$$

but in practice, only the atomic orbital coefficients $c_{n, j}$ are specified, not the atomic orbitals $\varphi^{0}\left(\mathbf{r}-\mathbf{R}_{j}\right)$ themselves. One solution would be to simply choose a type of atomic orbital (e.g., a Gaussian or a Slater orbital), but then the results will depend on an arbitrarily chosen extinction exponent. More rigorous would be to take the atomic orbital in the limit of high localization since this is in line with the HL approximation: neglect of overlap and only consideration of nearest-neighbors interactions. This would of course lead to numerical problems when evaluating the gradient of phase. Therefore we derive below the topological vorticity along a closed path of edges.

On the bond connecting the atoms s and t, only the atomic orbitals on s and t will contribute to $\psi_{n}$

$$
\begin{gathered}
\psi_{n}\left(\mathbf{r}^{\prime}\right)=c_{n, \mathrm{~s}} \exp \left(-i \frac{2 \pi}{\phi_{0}} \mathbf{A}\left(\mathbf{R}_{\mathrm{s}}\right) \cdot \mathbf{r}^{\prime}\right) \varphi^{0}\left(\mathbf{r}^{\prime}-\mathbf{R}_{\mathrm{s}}\right)+ \\
c_{n, \mathrm{t}} \exp \left(-i \frac{2 \pi}{\phi_{0}} \mathbf{A}\left(\mathbf{R}_{\mathrm{t}}\right) \cdot \mathbf{r}^{\prime}\right) \varphi^{0}\left(\mathbf{r}^{\prime}-\mathbf{R}_{\mathrm{t}}\right) \\
\mathbf{r}^{\prime}=\overline{\mathbf{R}}_{\mathrm{st}}^{\mathrm{g}}+f \Delta \mathbf{R}_{\mathrm{st}}, \quad-1 / 2 \leq f \leq 1 / 2
\end{gathered}
$$

where $\mathbf{r}^{\prime}$ can only vary along the bond. Since $\mathbf{A}\left(\mathbf{R}_{\mathrm{s}}\right) \cdot f \Delta \boldsymbol{R}_{\mathrm{st}}=$ $\mathbf{A}\left(\boldsymbol{R}_{\mathrm{t}}\right) \cdot f \Delta \boldsymbol{R}_{\mathrm{st}}=\mathbf{A}\left(\overline{\mathbf{R}}_{\mathrm{st}}^{\mathrm{g}}\right) \cdot f \Delta \boldsymbol{R}_{\mathrm{st}}=f \phi_{\mathrm{st}}$, we can simplify it to

$$
\begin{aligned}
& \psi_{n}\left(\mathbf{r}^{\prime}\right)=\exp \left(-i f \frac{2 \pi \phi_{\mathrm{st}}}{\phi_{0}}\right) \times \\
& \left\{\left|c_{n, \mathrm{~s}}\right| \exp \left(i\left\{\gamma_{n, \mathrm{~s}}-\frac{2 \pi}{\phi_{0}} \mathbf{A}\left(\mathbf{R}_{\mathrm{s}}\right) \cdot \overline{\mathbf{R}}_{\mathrm{st}}^{\mathrm{g}}\right\}\right) \varphi^{0}\left((f+1 / 2) \Delta \boldsymbol{R}_{\mathrm{st}}\right)+\right. \\
& \left.\left|c_{n, \mathrm{t}}\right| \exp \left(i\left\{\gamma_{n, \mathrm{t}}-\frac{2 \pi}{\phi_{0}} \mathbf{A}\left(\mathbf{R}_{\mathrm{t}}\right) \cdot \mathbf{R}_{\mathrm{st}}^{\mathrm{g}}\right\}\right) \varphi^{0}\left((f-1 / 2) \Delta \boldsymbol{R}_{\mathrm{st}}\right)\right\}
\end{aligned}
$$

with $\gamma_{n, \mathrm{~s}}, \gamma_{n, \mathrm{t}}$ the phases of the coefficients $c_{n, \mathrm{~s}}$ and $c_{n, \mathrm{t}}$, respectively.

The line integral along the edge st, from atom $s$ to $t$, is equal to

$$
\begin{aligned}
& \int_{s}^{t} \operatorname{Im}\left[\nabla \ln \psi_{n}\left(\mathbf{r}^{\prime}\right)\right] \cdot \mathrm{d} \mathbf{r}^{\prime}= \\
& \operatorname{Im}\left[\int_{f=-1 / 2}^{1 / 2} \partial_{f} \ln \psi_{n}\left(\overline{\mathbf{R}}_{\mathrm{st}}^{\mathrm{g}}+f \Delta \boldsymbol{R}_{\mathrm{st}}\right) \mathrm{d} f\right]
\end{aligned}
$$

For the radial part of the atomic orbitals one can take any type of normalized function, as long as we take the limit of high

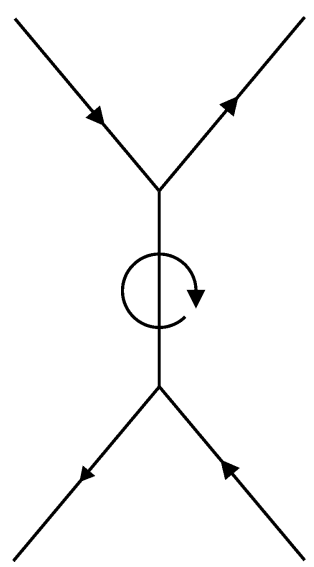

Figure 12. If a vortex is exactly located on an edge, its direction can be found from the neighboring edges.

localization in the end. By choosing a Gaussian, $\varphi^{0}\left(\mathbf{r}-\mathbf{R}_{j}\right) \sim$ $\exp \left(-b\left(\mathbf{r}-\mathbf{R}_{j}\right)^{2}\right)$, the integral can be solved analytically

$$
\begin{aligned}
\int_{f=-1 / 2}^{1 / 2} \partial_{f} \ln \psi_{n}\left(\overline{\mathbf{R}}_{\mathrm{st}}^{\mathrm{g}}+f \Delta \boldsymbol{R}_{\mathrm{st}}\right) \mathrm{d} f=-b \Delta R_{\mathrm{st}}{ }^{2}-i \frac{2 \pi \phi_{\mathrm{st}}}{\phi_{0}}- \\
\ln \frac{\left|c_{n, \mathrm{~s}}\right| \exp \left(i \gamma_{n, \mathrm{~s}}+b \Delta R_{\mathrm{st}}{ }^{2}\right)+\left|c_{n, \mathrm{t}}\right| \exp \left(i\left[\gamma_{n, \mathrm{t}}+\frac{2 \pi \phi_{\mathrm{st}}}{\phi_{0}}\right]\right)}{\left|c_{n, \mathrm{~s}}\right| \exp \left(i \gamma_{n, \mathrm{~s}}-b \Delta R_{\mathrm{st}}{ }^{2}\right)+\left|c_{n, \mathrm{t}}\right| \exp \left(i\left[\gamma_{n, \mathrm{t}}+\frac{2 \pi \phi_{\mathrm{st}}}{\phi_{0}}\right]\right)}
\end{aligned}
$$

which, by taking the limit of high localization, $b \rightarrow \infty$ and taking the imaginary part, reduces to

$$
\begin{aligned}
& \int_{s}^{t} \operatorname{Im}\left[\nabla \ln \psi_{n}(\mathbf{r})\right] \cdot \mathrm{d} \mathbf{l}=\Delta \tilde{\gamma}_{n, \mathrm{st}}= \\
& \Delta \gamma_{n, \mathrm{st}}-2 \pi \operatorname{Int}\left[\frac{\Delta \gamma_{n, \mathrm{st}}}{2 \pi}+\frac{\phi_{\mathrm{st}}}{\phi_{0}}\right]
\end{aligned}
$$

with $\Delta \gamma_{n, \mathrm{st}}=\gamma_{n, \mathrm{t}}-\gamma_{n, \mathrm{~s}}$. We call $\Delta \tilde{\gamma}_{n \text {,st }}$ the bond phase of an edge. The vorticity of a path is then obtained by summing all the bond phases of that path

$$
v=-\sum_{s t}^{C} \frac{\Delta \tilde{\gamma}_{n, \mathrm{st}}}{2 \pi}
$$

where the summation runs over the edges st of the closed path. When a vortex is located exactly on an edge, $\left(\Delta \gamma_{n, \mathrm{st}} / 2 \pi\right)+$ $\left(\phi_{\mathrm{st}} / \phi_{0}\right)$ is half-integer and hence the bond phase $\Delta \tilde{\gamma}_{n \text {,st }}$ of that edge is not unambiguously defined, which means that also the vorticity of any path containing that edge cannot be unambiguously determined. In many cases this can be solved by simply taking a slightly higher or lower flux such that the vortex moves away from the edge. However, when a vortex is fixed on the edge because of symmetry reasons, this cannot be done. In the cases we have encountered it, the direction of the vortex on the edge could be deduced logically from the bond phases on the neighboring edges (Figure 12). The vorticity of a path containing such an edge vortex depends then on the choice: should the edge vortex be included within the path or excluded.

\section{B. SNM Method}

B.1. Hamiltonian and Wave Function. Here we give the derivation of our screened nuclei approximation with magnetic field method (SNM). We investigate nanographenes subject to 
an external uniform magnetic field perpendicular to the plane of the molecule, i.e., $\mathbf{B}=B \mathbf{e}_{z}$. Neglecting the magnetic fields from nuclear spins, the vector potential $\mathbf{A}$ related to the magnetic field by $\mathbf{B}=\nabla \times \mathbf{A}$ reads in its simplest form

$$
\mathbf{A}(\mathbf{r})=\frac{B}{2} \mathbf{e}_{z} \times \mathbf{r}
$$

with $\mathbf{r}$ the coordinate vector. The choice of $\mathbf{A}$ is not unique since $\nabla \times(\mathbf{A}+\nabla u)=\nabla \times \mathbf{A}$, with $u(\mathbf{r})$ an arbitrary continuous scalar function. For such a choice of gauge the exact wave functions are only transformed by a phase factor $\exp \left(-i\left(2 \pi / \phi_{0}\right) u\right)$ while the physical observables are unchanged. Unfortunately, for finite basis sets the approximate wave functions and observables inevitably do depend nontrivially on the choice of gauge. However, several methods exist to remove the dependence on one specific class of gauge transformations, namely, the shift of origin of the vector potential: $u=$ $-\mathbf{r} \cdot \mathbf{A}\left(\mathbf{r}_{0}\right)$ so $\nabla u=-\mathbf{A}\left(\mathbf{r}_{0}\right)$. These methods can be divided into the discrete ${ }^{32-34}$ and the more recently developed continuous ${ }^{35,36}$ distribution of gauge origin methods. Of the former group is the gauge including atomic orbitals (GIAO) method ${ }^{34}$ the most common. This method, originally introduced by London, ${ }^{37}$ expresses the molecular orbitals $\psi_{n}$ as linear combinations of gauge including atomic orbitals $\varphi^{\mathrm{B}}$. Since the electronic properties of graphitic systems are mainly determined by the $\pi$-orbitals, we employ a minimal basis set of one $2 \mathrm{p}_{z}$ Slater orbital per atom

$$
\begin{gathered}
\psi_{n}(\mathbf{r})=\sum_{j} c_{n, j} \varphi^{\mathrm{B}}\left(\mathbf{r}-\mathbf{R}_{j}\right) \\
\varphi^{\mathrm{B}}\left(\mathbf{r}-\mathbf{R}_{j}\right)=\exp \left(-i \frac{2 \pi}{\phi_{0}} \mathbf{A}\left(\mathbf{R}_{j}\right) \cdot \mathbf{r}\right) \varphi^{0}\left(\mathbf{r}-\mathbf{R}_{j}\right) \\
\varphi^{0}(\mathbf{r})=\frac{1}{\sqrt{2 \pi}} \frac{1}{4}\left(\frac{Z_{\text {eff }}}{a_{0}}\right)^{5 / 2} z \exp \left(-\frac{Z_{\text {eff }}}{2 a_{0}} r\right)
\end{gathered}
$$

where $c_{n, j}$ denotes the atomic orbital coefficients, $\varphi^{\mathrm{B}}$ and $\varphi^{0}$ are the Slater orbitals with and without gauge included, and $Z_{\text {eff }}$ is the effective charge of the carbon atom, treated here as an adjustable parameter. To ensure rapid analytical calculation of the Hamiltonian matrix elements, the Slater orbital is accurately fitted to four Gaussian orbitals.

Solving the Fock matrix equation

$$
\begin{gathered}
(\mathbf{H}-\epsilon \mathbf{S}) \mathbf{C}=0 \\
\mathbf{H}=\mathbf{T}+\mathbf{V}
\end{gathered}
$$

requires knowledge of the overlap integrals $S_{\text {st }}$, kinetic energy integrals $T_{\text {st }}$, and potential energy integrals $V_{\text {st }}$ which have the following general form

$$
\begin{gathered}
S_{\mathrm{st}}=\int \mathrm{e}^{i 2 \pi / \phi_{0} \mathbf{A}\left(\Delta \boldsymbol{R}_{\mathrm{st}}\right) \cdot \mathbf{r}} \varphi^{0}\left(\mathbf{r}-\mathbf{R}_{\mathrm{s}}\right) \varphi^{0}\left(\mathbf{r}-\mathbf{R}_{\mathrm{t}}\right) \mathrm{d} \tau \\
T_{\mathrm{st}}=-\frac{\hbar^{2}}{2 m} \int \mathrm{e}^{i 2 \pi / \phi_{0} \mathbf{A}\left(\Delta \boldsymbol{R}_{\mathrm{st}}\right) \cdot \mathbf{r}} \varphi^{0}\left(\mathbf{r}-\mathbf{R}_{\mathrm{s}}\right) \times \\
{\left[\nabla+i \frac{2 \pi}{\phi_{0}} \mathbf{A}\left(\mathbf{r}-\mathbf{R}_{\mathrm{t}}\right)\right]^{2} \varphi^{0}\left(\mathbf{r}-\mathbf{R}_{\mathrm{t}}\right) \mathrm{d} \tau} \\
V_{\mathrm{st}}=\int \mathrm{e}^{i 2 \pi / \phi_{0} \mathbf{A}\left(\Delta \boldsymbol{R}_{\mathrm{st}}\right) \cdot \mathbf{r}} \varphi^{0}\left(\mathbf{r}-\mathbf{R}_{\mathrm{s}}\right) V(\mathbf{r}) \varphi^{0}\left(\mathbf{r}-\mathbf{R}_{\mathrm{t}}\right) \mathrm{d} \tau
\end{gathered}
$$

with $\Delta \boldsymbol{R}_{\mathrm{st}}=\mathbf{R}_{\mathrm{s}}-\mathbf{R}_{\mathrm{t}}$. Note that within HL theory, ${ }^{21}$ the second term between brackets in eq 60 is neglected and that for all three integrals, the approximation

$$
\begin{aligned}
& \int \mathrm{e}^{i 2 \pi / \phi_{0} \mathbf{A}\left(\Delta \boldsymbol{R}_{\mathrm{st}}\right) \cdot \mathbf{r}} \varphi^{0}\left(\mathbf{r}-\mathbf{R}_{\mathrm{s}}\right) \hat{O} \varphi^{0}\left(\mathbf{r}-\mathbf{R}_{\mathrm{t}}\right) \mathrm{d} \tau \approx \\
& \mathrm{e}^{i 2 \pi / \phi_{0} \mathbf{A}\left(\Delta \boldsymbol{R}_{\mathrm{st}}\right) \cdot \overline{\mathbf{R}}_{\mathrm{st}} \mathrm{g}} \int \varphi^{0}\left(\mathbf{r}-\mathbf{R}_{\mathrm{s}}\right) \hat{O} \varphi^{0}\left(\mathbf{r}-\mathbf{R}_{\mathrm{t}}\right) \mathrm{d} \tau
\end{aligned}
$$

is made. Closed form formulas for the expressions (59) and (60) are given in Appendix B.2, with the atomic orbitals $\varphi^{0}$ expressed as arbitrary linear combinations of Gaussian orbitals. The potential energy $V$ includes both electron-nucleus and electron-electron interactions. The electron-nucleus interaction can be expressed analytically (see Appendix B.2), but the electron-electron interaction-in for instance a density functional or Hartree-Fock approach-should be solved iteratively and requires the calculation of a large number of integrals. To circumvent these problems, the potential energy is approximated by a system of screened nuclei ${ }^{38-41}$

$$
V(\mathbf{r})=-\sum_{j=1}^{N} \frac{Z_{\mathrm{eff}} e}{4 \pi \epsilon_{0} r} \chi\left(\mathbf{r}-\mathbf{R}_{j}\right)
$$

where the screening function $\chi(\mathbf{r})$ should obey the boundary conditions $\chi(0)=1$ and $\chi(\infty)=0$. The solution of (61), within the approximation described by eq 63, is given in Appendix B.2, under the assumption that $\chi(\mathbf{r})$ can be expressed as a linear combination of Gaussians. We found that by expressing $\chi(\mathbf{r})$ by a single Gaussian, i.e., $\chi(\mathbf{r})=\exp \left(-\alpha r^{2}\right)$, already good results were obtained.

The two outer limits of the nanographenes are the infinite graphite sheet on one hand and benzene on the other hand. In ref 42, an analytical formula reproducing the DFT band spectrum of the graphite sheet with excellent precision was given. The two parameters of our model, $Z_{\mathrm{eff}}$ and $\alpha$, were fitted to match this band spectrum as close as possible. ${ }^{43}$ This resulted in $Z_{\text {eff }}=2.9$ and $\alpha=1.7 \AA^{-2}$. Applied to benzene, the model gives a value of $5.6 \mathrm{eV}$ for the HOMO-LUMO gap, which compares well with the empirical value of around $5 \mathrm{eV}^{44}$ and gives confidence in the method.

B.2. Integrals of the SNM Method. Here we derive the closed form formulas for the overlap integrals, kinetic energy integrals, and potential energy integrals encountered in the SNM method. Under the assumption that the gauge including atomic $2 \mathrm{p}_{z}$ orbital can be described by a set of gauge including normalized Gaussian orbitals

$$
\begin{gathered}
\varphi^{\mathrm{B}}\left(\mathbf{r}-\mathbf{R}_{\mathrm{s}}\right) \approx \sqrt{\frac{1}{A}} \sum_{k} d_{k} g_{k}^{\mathrm{B}}\left(\mathbf{r}-\mathbf{R}_{\mathrm{s}}\right) \\
g_{k}^{\mathrm{B}}\left(\mathbf{r}-\mathbf{R}_{\mathrm{s}}\right)=\exp \left(-i \frac{2 \pi}{\phi_{0}} \mathbf{A}\left(\mathbf{R}_{\mathrm{s}}\right) \cdot \mathbf{r}\right) g_{k}^{0}\left(\mathbf{r}-\mathbf{R}_{\mathrm{s}}\right) \\
g_{k}{ }^{0}\left(\mathbf{r}-\mathbf{R}_{\mathrm{s}}\right)=\frac{2^{7 / 4} b_{k}^{5 / 4}}{\pi^{3 / 4}} z \exp \left(-b_{k}\left(\mathbf{r}-\mathbf{R}_{\mathrm{s}}\right)^{2}\right)
\end{gathered}
$$

with $A$ a norm factor

$$
A=\sum_{k, l} d_{k} d_{l}\left(\frac{4 b_{k} b_{l}}{\left(b_{k}+b_{l}\right)^{2}}\right)^{5 / 4}
$$


A matrix element of the overlap, kinetic energy or potential energy matrix, between two $2 \mathrm{p}_{z}$ orbitals located at respectively $\mathbf{R}_{\mathrm{s}}$ and $\mathbf{R}_{\mathrm{t}}$ can then be written as

$$
\begin{gathered}
\left\langle\varphi^{\mathrm{B}}\left(\mathbf{r}-\mathbf{R}_{\mathrm{s}}\right)|\hat{O}| \varphi^{\mathrm{B}}\left(\mathbf{r}-\mathbf{R}_{\mathrm{t}}\right)\right\rangle= \\
\frac{1}{A} \sum_{k, l} d_{k} d_{l}\left\langle g_{k}^{\mathrm{B}}\left(\mathbf{r}-\mathbf{R}_{\mathrm{s}}\right)|\hat{O}| g_{l}^{\mathrm{B}}\left(\mathbf{r}-\mathbf{R}_{\mathrm{t}}\right)\right\rangle \\
\hat{O}=1,-\frac{\mathrm{p}^{2}}{2 m}\left[\nabla+i \frac{2 \pi}{\phi_{0}} \mathbf{A}\left(\mathbf{r}-\mathbf{R}_{j}\right)\right]^{2} \text { or } V
\end{gathered}
$$

We can thus focus on the matrix element between two gauge including Gaussians. Defining $\Delta \boldsymbol{R}_{\mathrm{st}}=\mathbf{R}_{\mathrm{t}}-\mathbf{R}_{\mathrm{s}}$ and a weighted center $\overline{\mathbf{R}}_{\mathrm{st}}^{\mathrm{w}}=\left(b_{k} \mathbf{R}_{\mathrm{s}}+b_{l} \mathbf{R}_{\mathrm{t}}\right) /\left(b_{k}+b_{l}\right)$, one gets

$$
\begin{aligned}
&\left\langle g_{k}^{\mathrm{B}}\left(\mathbf{r}-\mathbf{R}_{\mathrm{s}}\right)|\hat{O}| g_{l}^{\mathrm{B}}\left(\mathbf{r}-\mathbf{R}_{\mathrm{t}}\right)\right\rangle= \\
&\left\langle g_{k}^{\mathrm{B}}\left(\mathbf{r}+\overline{\mathbf{R}}_{\mathrm{st}}^{\mathrm{w}}-\mathbf{R}_{\mathrm{s}}\right)|\hat{O}| g_{l}^{\mathrm{B}}\left(\mathbf{r}+\overline{\mathbf{R}}_{\mathrm{st}}^{\mathrm{w}}-\mathbf{R}_{\mathrm{t}}\right)\right\rangle= \\
& \mathrm{e}^{i 2 \pi / \phi_{0} B \int_{\mathrm{st}}} \int \mathrm{e}^{-i 2 \pi / \phi_{0} \mathbf{A}\left(\Delta \boldsymbol{R}_{0}\right) \cdot \mathbf{r}} \\
& \quad g_{k}{ }^{0}\left(\mathbf{r}+\frac{b_{l} \Delta \boldsymbol{R}_{\mathrm{st}}}{b_{k}+b_{l}}\right) \hat{O} g_{l}{ }^{0}\left(\mathbf{r}-\frac{b_{k} \Delta \boldsymbol{R}_{\mathrm{st}}}{b_{k}+b_{l}}\right) \mathrm{d} \tau
\end{aligned}
$$

It is worth noting that the factor $\mathrm{e}^{i 2 \pi / \phi_{0} B \int_{\text {st }}}$ is also present in the elements of the HL matrix, where it forms the only magneticdependent ingredient of the theory.

The overlap and kinetic energy matrix elements can be found by standard integration techniques. For the overlap element one has

$$
\begin{aligned}
& \left\langle g_{k}^{\mathrm{B}}\left(\mathbf{r}-\mathbf{R}_{\mathrm{s}}\right) \mid g_{l}^{\mathrm{B}}\left(\mathbf{r}-\mathbf{R}_{\mathrm{t}}\right)\right\rangle= \\
& \quad\left(\frac{4 b_{k} b_{l}}{\left(b_{k}+b_{l}\right)^{2}}\right)^{5 / 4} \mathrm{e}^{i 2 \pi / \phi_{0} B \rho_{\mathrm{st}}} \exp \left(-\frac{\left(\Delta R_{\mathrm{st}}\right)^{2}}{b_{k}+b_{l}}\left(b_{k} b_{l}+\frac{1}{16}\left(\frac{2 \pi B}{\phi_{0}}\right)^{2}\right)\right)
\end{aligned}
$$

while the kinetic energy matrix element is equal to

$$
\begin{gathered}
-\frac{\hbar^{2}}{2 m}\left|g_{k}^{\mathrm{B}}\left(\mathbf{r}-\mathbf{R}_{\mathrm{s}}\right)\right|\left[\nabla+i \frac{2 \pi}{\phi_{0}} \mathbf{A}\left(\mathbf{r}-\mathbf{R}_{j}\right)\right]^{2}\left|g_{l}^{\mathrm{B}}\left(\mathbf{r}-\mathbf{R}_{\mathrm{t}}\right)\right\rangle= \\
\frac{\hbar^{2}}{2 m}\left\langle g_{k}^{\mathrm{B}}\left(\mathbf{r}-\mathbf{R}_{\mathrm{s}}\right) \mid g_{l}^{\mathrm{B}}\left(\mathbf{r}-\mathbf{R}_{\mathrm{t}}\right)\right\rangle\left[\frac{4 b_{k} b_{l}}{b_{k}+b_{l}}\left(\frac{5}{2}-\frac{b_{k} b_{l}}{b_{k}+b_{l}}\left(\Delta R_{\mathrm{st}}\right)^{2}\right)+\right. \\
\frac{1}{4}\left(\frac{1}{b_{k}+b_{l}}+\frac{b_{k}^{2}+b_{l}^{2}}{\left(b_{k}+b_{l}\right)^{2}}\left(\Delta R_{\mathrm{st}}\right)^{2}\right)\left(\frac{2 \pi B}{\phi_{0}}\right)^{2}- \\
\left.\frac{\left(\Delta R_{\mathrm{st}}\right)^{2}}{64\left(b_{k}+b_{l}\right)^{2}}\left(\frac{2 \pi B}{\phi_{0}}\right)^{4}\right]
\end{gathered}
$$

Hence apart from the modulation $\mathrm{e}^{i 2 \pi / \phi_{0} B \int_{\mathrm{st}}}$, also the size of these matrix elements varies with the magnetic field.

The potential energy matrix elements are derived under the assumption that the potential can be approximated by a system of screened nuclei and that the screening function can be represented by a sum of Gaussians.

$$
\begin{gathered}
V(\mathbf{r})=\sum_{j=1}^{N}-\frac{Z_{\mathrm{eff}} e}{4 \pi \epsilon_{0} r} \chi\left(\mathbf{r}-\mathbf{R}_{j}\right) \\
\chi(\mathbf{r})=\sum_{u} d_{\mathrm{u}} \exp \left(-\alpha_{u} r^{2}\right), \quad \sum_{u} d_{\mathrm{u}}=1
\end{gathered}
$$

The potential energy matrix element between two Gaussians can then be set equal to

$$
\begin{aligned}
& \left\langle g_{k}^{\mathrm{B}}\left(\mathbf{r}-\mathbf{R}_{\mathrm{s}}\right)|V| g_{l}^{\mathrm{B}}\left(\mathbf{r}-\mathbf{R}_{\mathrm{t}}\right)\right\rangle=-\frac{Z_{\mathrm{eff}} e}{4 \pi \epsilon_{0}} \times \\
& \sum_{j=1}^{N} \sum_{u} d_{\mathrm{u}}\left\langle g_{k}^{\mathrm{B}}\left(\mathbf{r}-\mathbf{R}_{\mathrm{s}}\right)\left|\frac{\exp \left(-\alpha_{\mathrm{u}}\left|r-\mathbf{R}_{j}\right|^{2}\right)}{\left|r-\mathbf{R}_{j}\right|}\right| g_{l}^{\mathrm{B}}\left(\mathbf{r}-\mathbf{R}_{\mathrm{t}}\right)\right\rangle
\end{aligned}
$$

The terms in the summation can be solved analytically

$$
\begin{aligned}
& \left\langle g_{k}^{\mathrm{B}}\left(\mathbf{r}-\mathbf{R}_{\mathrm{s}}\right)\left|\frac{\exp \left(-\alpha_{\mathrm{u}}\left|r-\mathbf{R}_{\mathrm{j}}\right|^{2}\right)}{\left|r-\mathbf{R}_{j}\right|}\right| g_{l}^{\mathrm{B}}\left(\mathbf{r}-\mathbf{R}_{\mathrm{t}}\right)\right\rangle= \\
& \left\langle g_{k}^{\mathrm{B}}\left(\mathbf{r}-\mathbf{R}_{\mathrm{s}}\right) \mid g_{l}^{\mathrm{B}}\left(\mathbf{r}-\mathbf{R}_{\mathrm{t}}\right)\right\rangle \frac{\left(b_{k}+b_{l}\right)^{5 / 2}}{\left(b_{k}+b_{l}+\alpha_{\mathrm{u}}\right)^{2}} \exp \left(-\frac{\alpha_{\mathrm{u}}}{b_{k}+b_{l}} Q\right) \times \\
& Q^{-1}\left(\frac{1}{\sqrt{\pi}} \exp (-Q)+\left(-\frac{1}{2} Q^{-1 / 2}+Q^{1 / 2}\right) \operatorname{Erf}\left[Q^{1 / 2}\right]\right)
\end{aligned}
$$

with $\operatorname{Erf}[z]$ the error function, defined as

$$
\operatorname{Erf}[z]=\frac{2}{\sqrt{\pi}} \int_{0}^{z} \mathrm{e}^{-t^{2}} \mathrm{~d} t
$$

and the complex quantity $Q$ given by

$$
\begin{aligned}
& Q= \\
& \frac{1}{b_{k}+b_{l}+\alpha_{\mathrm{u}}}\left[\left(b_{k}+b_{l}\right)\left(\mathbf{R}_{j}-\overline{\mathbf{R}}_{\mathrm{st}}^{\mathrm{w}}\right)+i \frac{1}{4} \frac{2 \pi B}{\phi_{0}}\left[\mathbf{e}_{z} \times\left(\Delta \mathbf{R}_{\mathrm{st}}\right)\right]\right]^{2}
\end{aligned}
$$

To obtain the unscreened electron-nucleus interaction, it is sufficient to put $\alpha_{\mathrm{u}}=0$ in eq 72 .

\section{References and Notes}

(1) Abrikosov, A. A. Fundamentals of the Theory of Metals; NorthHolland: Amsterdam, 1988.

(2) de Gennes, P.-G. Superconductivity of Metals and Alloys; Benjamin: New York, 1966.

(3) Chibotaru, L. F.; Ceulemans, A.; Bruyndoncx, V.; Moshchalkov, V. V. Nature 2000, 408, 833-835.

(4) Chibotaru, L. F.; Ceulemans, A.; Morelle, M.; Teniers, G.; Carballeira, C.; Moshchalkov, V. V. J. Math. Phys. 2005, 46, 095108.

(5) Chibotaru, L. F.; Ceulemans, A.; Lorenzini, M.; Moshchalkov, V. V. Europhys. Lett. 2003, 63, 159-165.

(6) Dirac, P. A. Proc. R. Soc. London, Ser. A 1931, 133, 60

(7) Riess, J. Phys. Rev. D 1970, 2, 647-653.

(8) Bialynicki-Birula, I.; Bialynicka-Birula, Z. Phys. Rev. D 1971, 3, $2410-2412$

(9) Hirschfelder, J. O.; Christoph, A. C.; Palke, W. E. J. Chem. Phys. 1974, 61, 5435-5455.

(10) Hirschfelder, J. O.; Goebel, C. J.; Bruch, L. W. J. Chem. Phys. 1974, 61, 5456-5459.

(11) Heller, D. F.; Hirschfelder, J. O. J. Chem. Phys. 1977, 66, 19291933

(12) Hirschfelder, J. O. J. Chem. Phys. 1978, 67, 5477-5483.

(13) Gomes, J. A. N. F. J. Chem. Phys. 1983, 78, 3133-3139.

(14) Soncini, A.; Fowler, P. W. Chem. Phys. Lett. 2004, 400, 213220

(15) Dotz, F.; Brand, J. D.; Ito, S.; Gherghel, L.; Müllen, K. J. Am Chem. Soc. 2000, 122, 7707-7717.

(16) Grimsdale, A. C.; Müllen, K. Angew. Chem., Int. Ed. 2005, 44 $5592-5629$

(17) Samori, P.; Severin, N.; Simpson, C. D.; Müllen, K.; Rabe, J. P. J. Am. Chem. Soc. 2002, 124, 9454-9457.

(18) Compernolle, S.; Chibotaru, L. F.; Ceulemans, A. Chem. Phys. Lett. 2006, 428, 119-124.

(19) Pasquarello, A.; Schluter, M.; Haddon, R. C. Phys. Rev. A 1993, $47,1783-1789$

(20) Ceulemans, A.; Chibotaru, L. F.; Fowler, P. W. Phys. Rev. Lett. 1998, 80, 1861-1864. 
(21) Pople, J. A. J. Chem. Phys 1962, 37, 53-59.

(22) Corey, E. J.; Petersson, G. A. J. Am. Chem. Soc. 1972, 94, 460465.

(23) Ceulemans, A.; Fowler, P. W. J. Chem. Soc., Faraday Trans. 1995, 91, 3089-3093.

(24) Salem, L. The molecular orbital theory of conjugated systems; Benjamin: New York, 1966.

(25) Haddon, R. C. J. Am. Chem. Soc. 1979, 101, 1722-1728.

(26) Kobayashi, Y.; Fukui, K.-I.; Enoki, T.; Kusakabe, K.; Kaburagi, Y. Phys. Rev. B 2005, 71, 193406.

(27) Simpson, C. D.; Brand, J. D.; Berresheim, A. J.; Przybilla, L.; Rader, H. J.; Müllen, K. Chem. Eur. J. 2002, 8, 1424-1429.

(28) Wang, Z.; Tomovic, Z.; Kastler, M.; Pretsch, R.; Negri, F.; Enkelmann, V.; Müllen, K. J. Am. Chem. Soc. 2004, 126, 7794-7795.

(29) Clar, E. J. Polycyclic Hydrocarbons; Academic: New York, 1964; Vol. 1

(30) Tyutyulkov, N.; Madjarova, G.; Dietz, F.; Müllen, K. J. Phys. Chem. B 1998, 102, 10183-10189.

(31) Stein, S. E.; Brown, R. L. J. Am. Chem. Soc. 1987, 109, 37213729 .
(32) Kutzelnigg, W. Isr. J. Chem. 1980, 19, 193-200.

(33) Schlinder, M.; Kutzelnigg, W. J. Chem. Phys. 1982, 76, 19191933.

(34) Ditchfield, R. Mol. Phys. 1974, 27, 789-807.

(35) Keith, T.; Bader, R. F. W. Chem. Phys. Lett. 1993, 210, 223-231.

(36) Coriani, S.; Lazzeretti, P.; Malagoli, M.; Zanasi, R. Theor. Chim. Acta 1994, 89, 181-192.

(37) London, F. J. Phys. Radium 1937, 8, 397-409.

(38) Dirac, P. A. M. Proc. Cambridge Philos. Soc. 1930, 26, 376-385.

(39) Thomas, L. H. Proc. Cambridge Philos. Soc. 1927, 23, 542-548.

(40) Fermi, E. Atti Accad. Naz. Lincei 1927, 6, 602.

(41) Fermi, E. Z. Phys. 1928, 48, 73-79.

(42) Reich, S.; Maultzsch, J.; Thomsen, C. Phys. Rev. B 2002, 66, 035412 .

(43) The reference band spectrum was produced with the third-neighbor tight-binding scheme of ref 42 , with the parameter set that reproduced accurately the band spectrum of graphene in the optical range as calculated by DFT with a double- $\zeta$, singly polarized basis set.

(44) Platt, J. R. J. Chem. Phys. 1947, 15, 419-420. 